\title{
Optimal vs. Robust: Applications to Planning Strategies Insights from a simulation study
}

Mladen Jovanović ${ }^{1}$ and Ivan Jukić 2

${ }^{1}$ Faculty of Sports and Physical Education, University of Belgrade, Serbia

${ }^{2}$ Sport Performance Research Institute New Zealand (SPRINZ), Auckland, New Zealand

\section{Corresponding author:}

Mladen Jovanović

Faculty of Sports and Physical Education

Blagoja Parovica 156

11030 Belgrade

Serbia

Email: coach.mladen.jovanovic@gmail.com

Twitter: physical_prep

Web: www.complementarytraining.net

\section{This document is a pre-print}

Version 3. Uploaded 01/04/2019

\section{Cite as:}

Jovanović, M. \& Jukić, I. (2019). Optimal vs. robust: applications to planning strategies. Insights from a simulation study. SportRxiv. doi: 10.31236/osf.io/8n4jf

DOI: $10.31236 /$ osf.io/8n4jf

All authors have read and approved this version of the manuscript for pre-print. 


\section{Abstract}

Traditional planning and periodization of training have been considered as one of the key elements when it comes to managing fatigue and maximizing both general and specific sports performance. However, these traditional concepts of periodization rely on the idea of designing an optimal training plan while assuming the stability of non-training factors. One of the most frequent logistical issues often seen in sports are missed sessions, both randomly (MCAR) and with a certain pattern (MNAR). Since these non-training factors considerably decrease the optimality of one periodization strategy, the objectives of this study were to assess the performance of different loading strategies for training load control and to explore what impact MCAR events had on the performance of these loading strategies. We simulated 19 different loading strategies. Each loading strategy was applied for 150 days, under normal conditions (no missing sessions), as well under MCAR conditions. The following MCAR conditions were simulated: (1) no missing days (missing 0 in 10), (2) missing 1 in 10, (3) missing 2 in 10, (4) missing 4 in 10, and (5) missing 6 in 10 days. Each scenario for every loading strategy was simulated 100 times. The performance was evaluated using an exponential moving average (EMA) approach to get acute training load and simple moving average (SMA) to get chronic training load (rolling 28 days), as well as acute to chronic workload ratio (ACWR) for every day in simulations. Moreover, between qualities standard deviation (btwnQualitiesSD) and ratio metric were introduced to estimate variance in training load between qualities and to determine the emphasis of a certain training quality compared total training load receptively. The novel 'don't break the chain' loading strategies demonstrated the most robust behavior under simulated MCAR conditions by the least drop in the total training load.

Keywords: periodization, simulation, randomization, robust, missing sessions, Markov chain 


\section{Introduction}

Contemporary planning and periodization of training (referred to as planning strategies in the current paper) relies on the idea that there is an optimal way to sequence training objectives, means and loads over different time frames ${ }^{1-8}$. Optimality of a particular approach or method, in terms of maximizing adaptation and skill acquisition while minimizing injury and overtraining risk, relies on many assumptions of predictability, as well as our ability to nominate potential outcomes and their probabilities ${ }^{9}$. For instance, individual and team needs are often based upon reductionist physiological and biomechanical perspectives ${ }^{10}$ which rely on a clearly defined hierarchy of qualities (constructs) that one needs to possess. Furthermore, responses and adaptations to the training intervention are often analyzed, using group-average-based statistical analysis ${ }^{11,12}$, which assumes ergodicity of the process involved, and application to a single individual ${ }^{13,14}$. This predictability and optimality, which are major assumptions of contemporary planning strategies, have recently been brought into question ${ }^{10,15-22}$.

Besides the psychological and biological complexities which influence individual training responses, logistical issues also affect planning strategies ${ }^{10}$. One common logistical issue is missed sessions. Missed sessions could occur entirely at random (missing completely at random or MCAR), or there might be a specific pattern (e.g., always missing Tuesdays, or lower body strength training days) which is commonly referred to as missing not at random (MNAR) ${ }^{23,24}$. Distinguishing between MCAR and MNAR events, and understanding the reasons behind MNAR events, can be considered an essential aspect of creating agile ${ }^{10}$ and individually tailored plans due to unpredictable situations often seen in sports and personal training. For example, during the in-season phase in team sports, speed and power work is commonly emphasized on a particular day between competitive games (usually third or fourth day after a game) that represents an optimal time frame to perform such an activity ${ }^{10}$. This optimal time assumes that athletes have recovered from the previous game at this point while leaving enough time before the next game for potential fatigue to dissipate. However, a problem may emerge if any player misses this workout, or the head coach suddenly changes her plans. In this hypothetical scenario, athletes will be without speed and power stimuli for approximately two weeks assuming no other direct speed and power work being performed during other sessions. Thus, next time an athlete performs speed and power work, there might be a sudden spike in training load which might represent potential cause in load-related issues such as excessive soreness or even injuries ${ }^{25}$. In this way, a training plan that is generally considered to be optimal can become harmful when circumstances change.

Considering these possible occurrences of MCAR and MNAR events in high-performance settings, which will increase uncertainty and therefore decrease optimality of the traditional approach to planning and periodization of training, more robust approaches are needed. Robustness has been defined as the degree to which a system or its component can function correctly in the presence of invalid inputs or stressful environmental conditions ${ }^{26}$. In a performance context, robust planning strategy can represent a particular way of planning that is resilient to unexpected changes (e.g., MCAR and MNAR events), lack of information, uncertainties, and changes in training context often seen when working with athletes. One potential and more robust strategy, that can overcome 
problems with regards to MCAR and MNAR events, might involve micro-dosing the work of different physical traits on consecutive days ${ }^{10}$. In this way, the risk of harm in the case of MCAR or MNAR events would be considerably reduced. Although this approach is robust to unpredictable changes, it lacks higher load saturation into a single quality that might be needed to progress certain quality forward in terms of adaptation ${ }^{6-8}$. Thus, there is a need for more complete planning strategies that are robust to uncertain conditions, but also allowing for training adaptations to occur through different loading strategies.

The objectives of this simulation study are to assess the performance of different planning strategies when facing MCAR events and to determine whether loading strategies cluster together using theoretical simulation in R statistical language 27.

\section{Methods}

\section{Loading strategies}

For the purpose of the current paper, five theoretical training qualities have been used as objectives of training. These qualities could represent physiological, individual or tactical skill as objectives (e.g., hypertrophy, maximum strength, maximal speed, aerobic endurance), or they might represent different training methods utilized in training system (e.g., maximal effort method, dynamic effort method, lactate threshold runs, sprinting). Development of these qualities is organized into fivedays microcycle (Figure 1), where each quality receives a total training load (expressed in arbitrary units, a.u.) of 100 points. Total training load during five-days microcycle is 500 a.u. The model assumes that qualities are independent of each other and that there are no residual effects nor interaction between qualities, loads performed, and days (i.e., the order of qualities development in a single session doesn't matter, nor the sequence matters). Although these are unrealistic assumptions in the real world, they are required for the theoretical simulation to allow behavior exploration and a comparison between different load planning strategies. 
Concentrated Loading Strategy

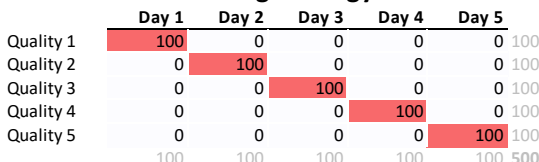

Hard-Easy Loading Strategy

\begin{tabular}{lrrrrr} 
& Day 1 & Day 2 & Day 3 & Day 4 & Day 5 \\
\cline { 2 - 6 } Quality 1 & 60 & 10 & 10 & 10 & 10 \\
Quality 2 & 10 & 60 & 10 & 10 & 10 \\
Quality 3 & 10 & 10 & 60 & 10 & 10 \\
Quality 4 & 10 & 10 & 10 & 60 & 10 \\
Quality 5 & 10 & 10 & 10 & 10 & 60
\end{tabular}

Increasing Loading Strategy

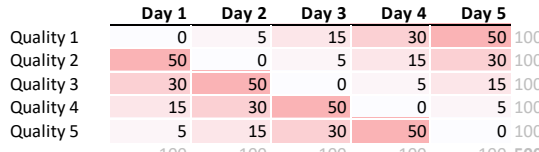

Ramp Decreasing Loading Strategy

\begin{tabular}{|c|c|c|c|c|c|}
\hline & Day 1 & Day 2 & Day 3 & Day 4 & Day 5 \\
\hline Quality 1 & 35 & 30 & 20 & 10 & 5 \\
\hline Quality 2 & 35 & 30 & 20 & 10 & 5 \\
\hline Quality 3 & 35 & 30 & 20 & 10 & 5 \\
\hline Quality 4 & 35 & 30 & 20 & 10 & 5 \\
\hline Quality 5 & 35 & 30 & 20 & 10 & 5 \\
\hline
\end{tabular}

Block Loading Strategy

\begin{tabular}{|l|r|r|r|r|r|} 
& Day 1 & Day 2 & Day 3 & Day 4 & Day 5 \\
\hline Quality 1 & 35 & 35 & 15 & 10 & 5 \\
\hline Quality 2 & 35 & 35 & 15 & 10 & 5100 \\
\hline Quality 3 & 35 & 35 & 15 & 10 & 5100 \\
\hline Quality 4 & 35 & 35 & 15 & 10 & 5100 \\
\hline Quality 5 & 35 & 35 & 15 & 10 & 5100
\end{tabular}

Microloading Strategy

\begin{tabular}{lrrrrr} 
& Day 1 & Day 2 & Day 3 & Day 4 & Day 5 \\
\cline { 2 - 6 } Quality 1 & 20 & 20 & 20 & 20 & 20 \\
Quality 2 & 20 & 20 & 20 & 20 & 20 \\
Quality 3 & 20 & 20 & 20 & 20 & 20 \\
Quality 4 & 20 & 20 & 20 & 20 & 20 \\
Quality 5 & 20 & 20 & 20 & 20 & 20
\end{tabular}

Medium-Easy Loading Strategy

\begin{tabular}{lrrrrr} 
& Day 1 & Day 2 & Day 3 & Day 4 & Day 5 \\
\cline { 2 - 6 } Quality 1 & 40 & 15 & 15 & 15 & 15 \\
Quality 2 & 15 & $\mathbf{4 0}$ & 15 & 15 & 15 \\
Quality 3 & 15 & 15 & 40 & 15 & 15 \\
Quality 4 & 15 & 15 & 15 & $\mathbf{4 0}$ & 15 \\
Quality 5 & 15 & 15 & 15 & 15 & $\mathbf{4 0}$
\end{tabular}

Decreasing Loading Strategy

\begin{tabular}{lrrrrr} 
& Day 1 & Day 2 & Day 3 & Day 4 & Day 5 \\
\cline { 2 - 6 } Quality 1 & 50 & 30 & 15 & 5 & 0 \\
Quality 2 & 0 & 50 & 30 & 15 & 51 \\
Quality 3 & 5 & 0 & 50 & 30 & 15 \\
\hline Quality 4 & 15 & 5 & 0 & 50 & 30 \\
\hline Quality 5 & 30 & 15 & 5 & 0 & 50
\end{tabular}

Ramp Increasing Loading Strategy

\begin{tabular}{|rrrrrr} 
& Day 1 & Day 2 & Day 3 & Day 4 & Day 5 \\
\cline { 2 - 6 } Quality 1 & 5 & 10 & 20 & 30 & 35 \\
\hline Quality 2 & 5 & 10 & 20 & 30 & 35 \\
\hline Quality 3 & 5 & 10 & 20 & 30 & 35 \\
\hline Quality 4 & 5 & 10 & 20 & 30 & 35 \\
\hline Quality 5 & 5 & 10 & 20 & 30 & 35
\end{tabular}

Barbell Loading Strategy*

\begin{tabular}{l|rrrrr} 
& Day 1 & Day 2 & Day 3 & Day 4 & Day 5 \\
\cline { 2 - 6 } Quality 1 & 35 & 41.25 & 10 & 10 & 10 \\
\hline Quality 2 & 35 & 10 & 41.25 & 10 & 10 \\
\hline Quality 3 & 35 & 10 & 10 & 41.25 & 10 \\
\hline Quality 4 & 35 & 10 & 10 & 10 & 41.25 \\
\hline Quality 5 & 35 & 10 & 10 & 10 & 10
\end{tabular}

* Days 2-5 are randomized

Figure 1. Loading strategies. Each one of five possible qualities receives 100 a.u. points per 5-day microcyle, and total training load in 5-day microcycle is equal to 500 a.u.

Figure 1 contains ten theoretical and archetypal training load planning strategies (referred to as loading strategies). Loading strategies are divided into four quadrants (Figure 2) using two criteria: (1) whether or not the daily training load sums up to 100 a.u. (flat loading and non-flat loading), and (2) whether or not the daily training load is equally distributed among five qualities (equally distributed and unequally distributed).

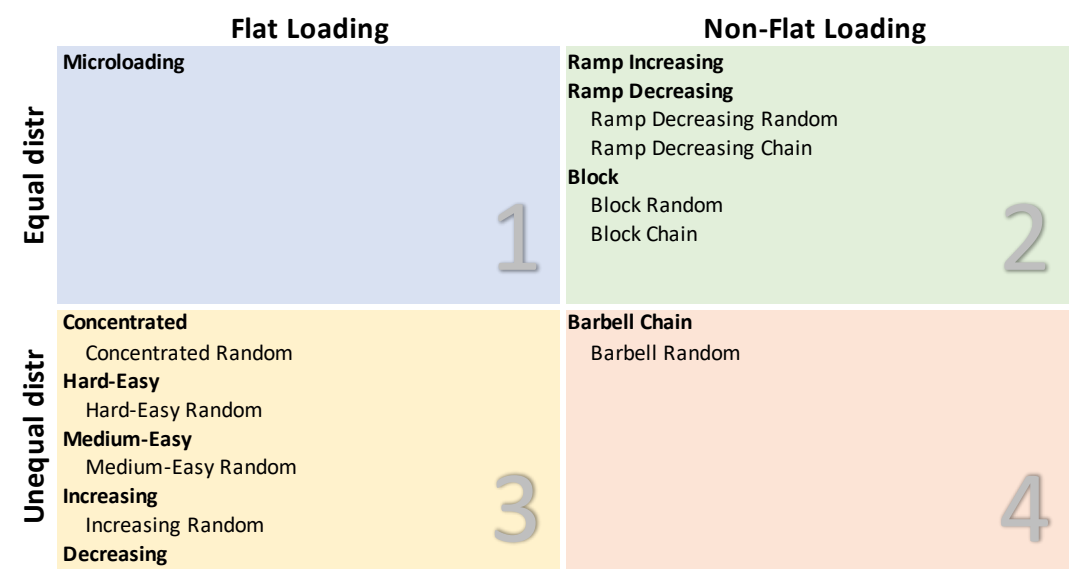

Figure 2. Loading strategies quadrant 
Figure 3 and Figure 4 contains graphical representations of the loading strategies over 15 days time span without any missing days.

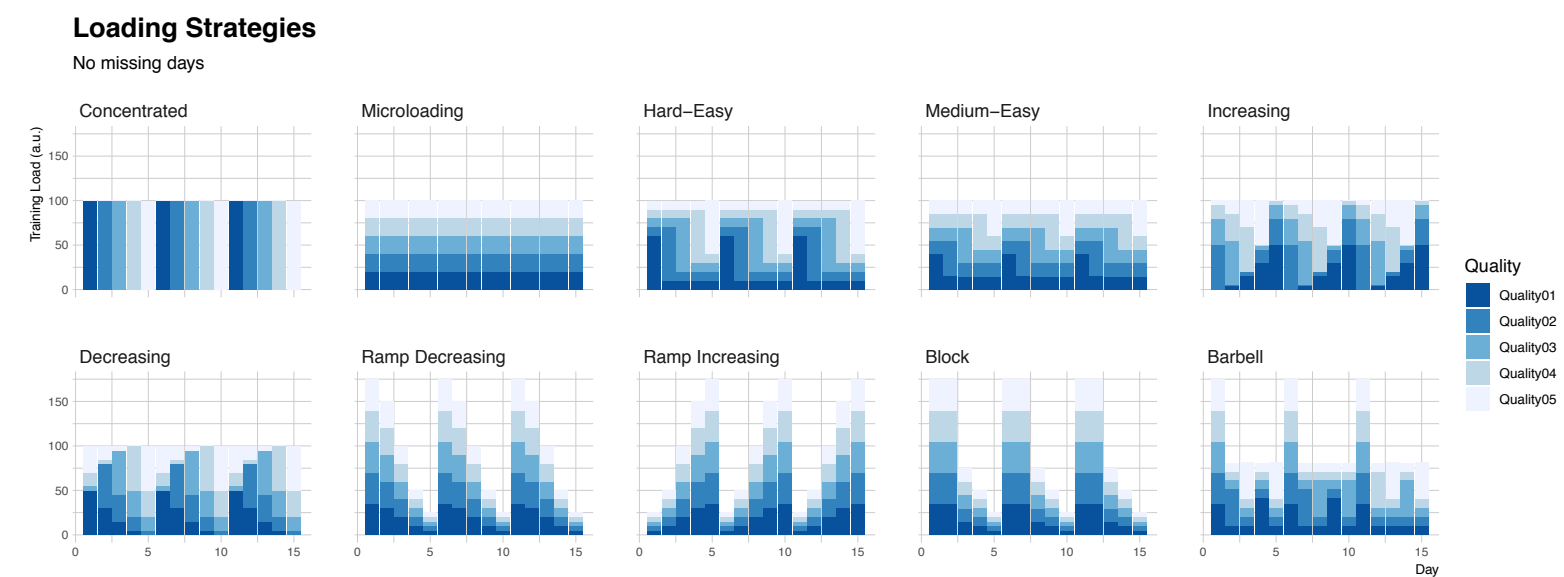

Figure 3. Stacked bar plot of loading strategies over 15 days without missing days

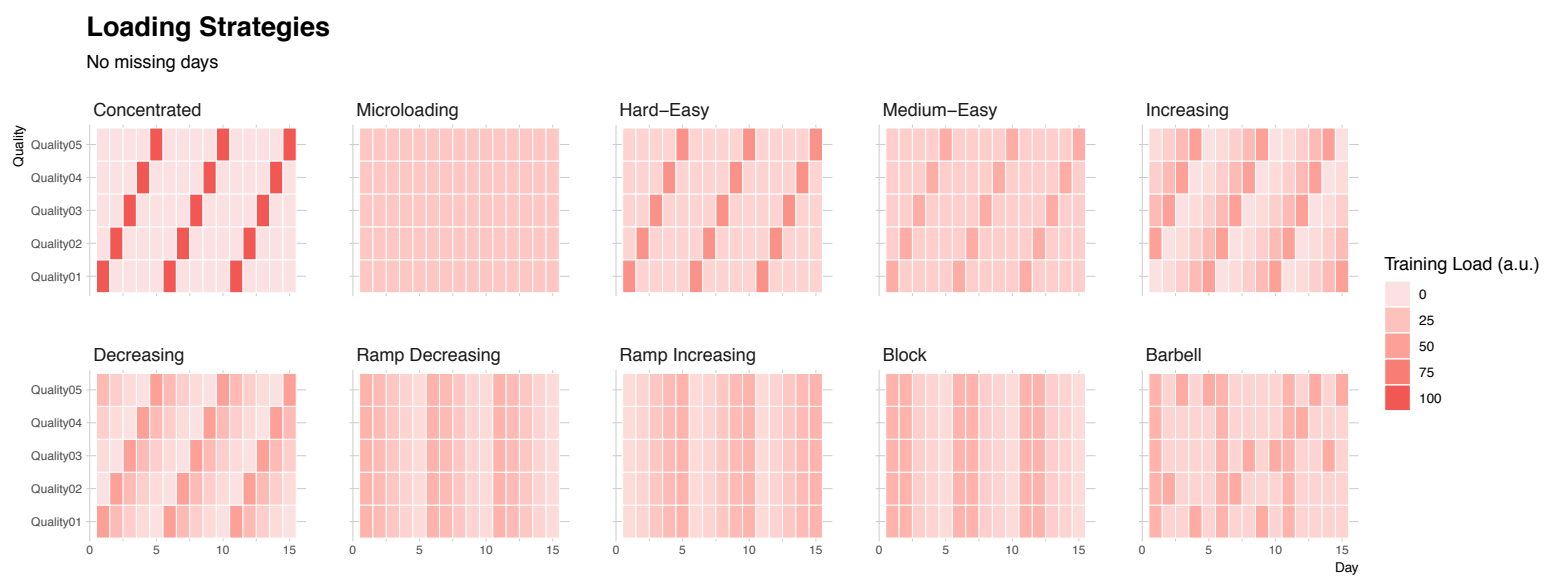

Figure 4. Tiles plot of loading strategies over 15 days without missing days

\section{Variants of loading strategies}

Based on the system behavior after a missing day occurs, three loading strategy variants were simulated: (1) normal, where loading strategy continued as nothing happened when missing day occurred (Figure 5), (2) random, where the order of days was randomized (Figure 6), and (3) don't break the chain modification (referred to as chain), where when a day is missed, the next day is restarted to day one (Figure 7). Making the first day in the chain strategies the hardest and ensuring coverage of all qualities is hypothesized to increase robustness in the face of MCAR conditions. 


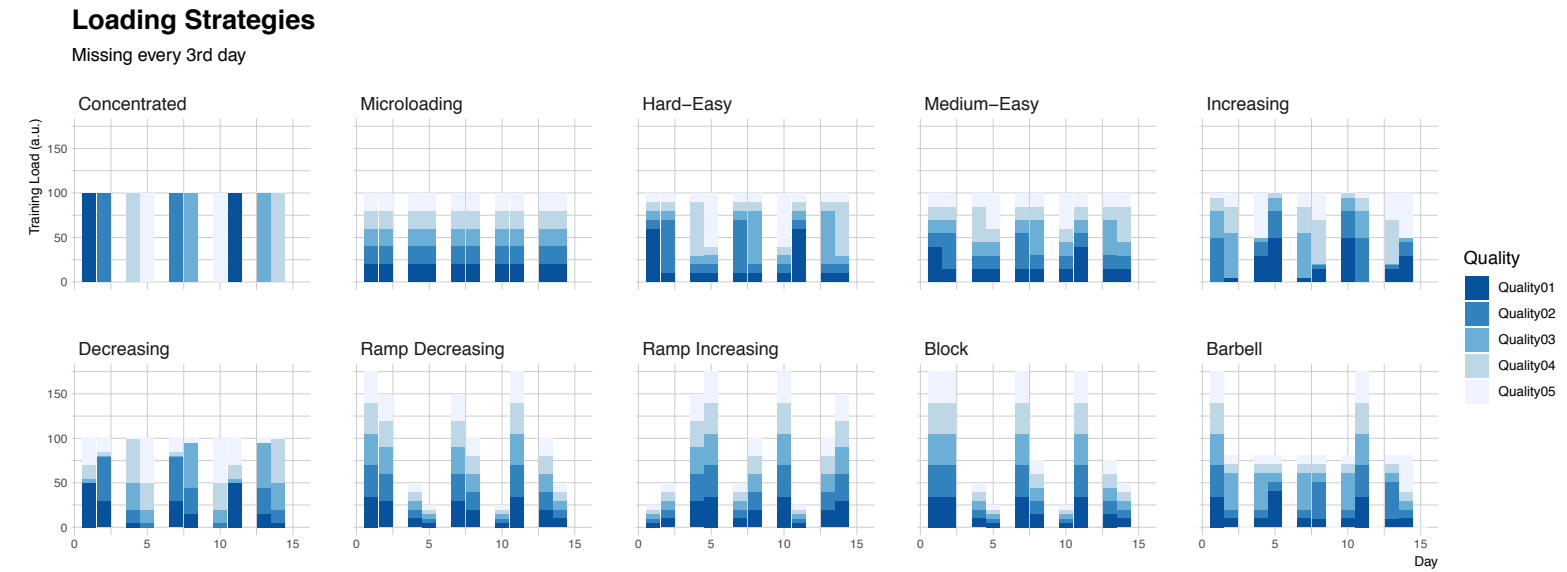

Figure 5. Stacked bar plot of loading strategies over 15 days with missing day every 3rd day.

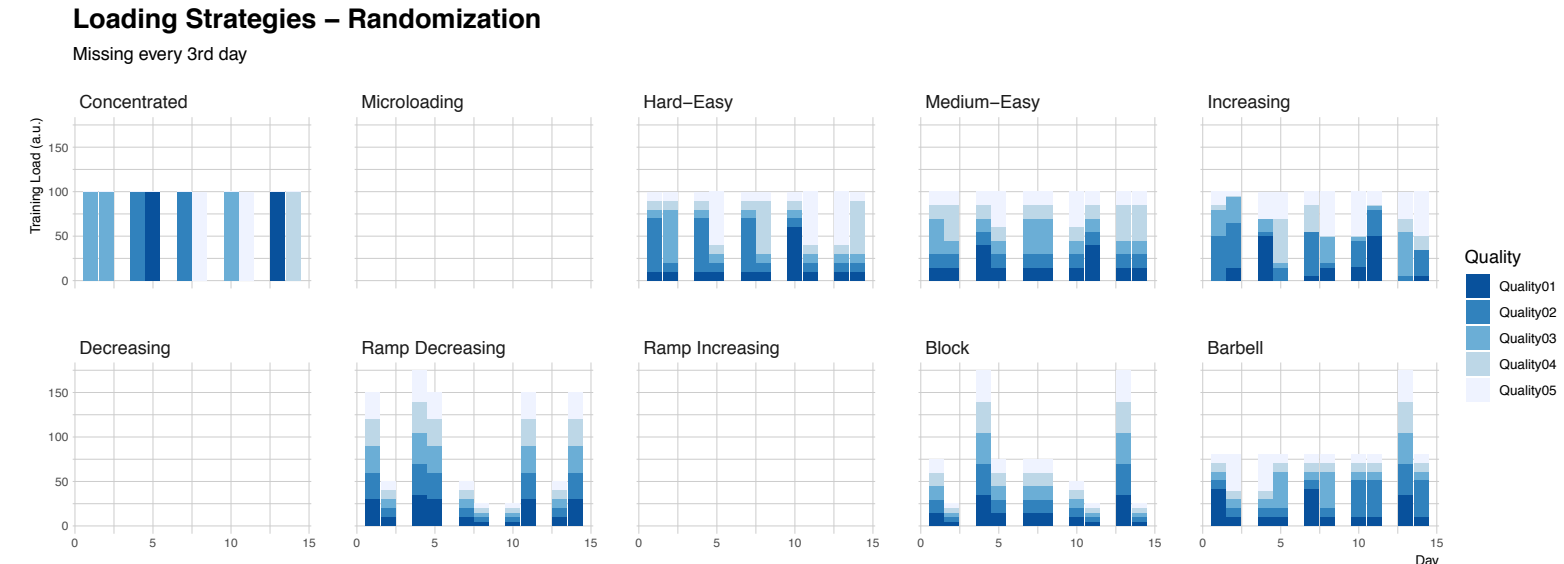

Figure 6. Stacked bar plot of loading strategies using randomization of day order over 15 days with missing day every 3rd day.

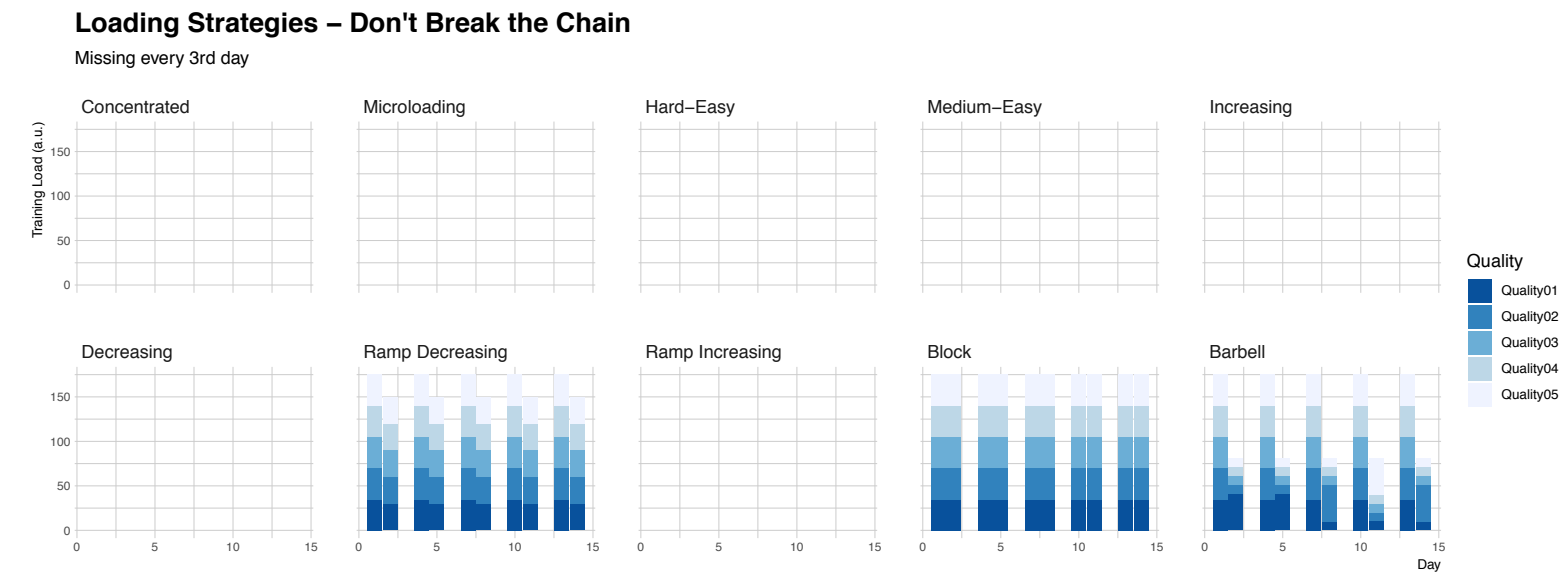

Figure 7. Stacked bar plot of loading strategies using 'don't break the chain' strategy over 15 days with missing day every 3rd day. 


\section{Barbell loading strategy}

Of the ten simulated strategies, one is unique: the barbell loading strategy (referred to as barbell). Barbell loading strategy represents a unique loading strategy influenced by Nassim Taleb's ${ }^{28}$ concept of decision making in uncertainty entitled barbell strategy ${ }^{10}$. According to Taleb, the barbell strategy consists of two parts: (1) protecting from the downside and (2) investing in the upside (Figure 8).

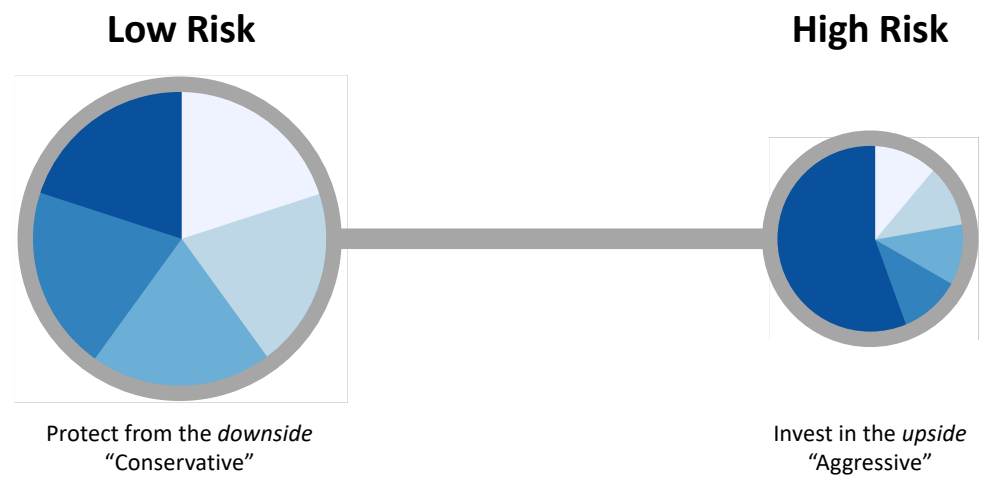

Figure 8. Barbell strategy. Consists of two asymmetrical parts: (1) protecting from the downside, and (2) investing in the upside. Adapted from Taleb ${ }^{28}$

Although the upside and downside concepts come from the investing domain, they have implications in training planning. From this perspective, downside represents negative consequences to performance, such as overtraining, undertraining, injury or boredom. Upside represents positive consequences to performance and adaptation. According to Taleb, one needs to make sure to protect from the downside first, before embracing the pursuit of any potential upside. Protection from the downside means making a training plan more robust, or resistant to perturbations and potential issues with someone being wrong in the first place. For example, one might erroneously conclude that to improve performance, an athlete must focus most of his training on improving Quality01 for the next month. However, this approach can unfortunately fail and bring down other essential qualities which further results in the time waste and decrease in performance. If this example succeeded, it would represent an upside example, but without protection from the downside in the first place, it represents a very fragile strategy. For this reason, optimal planning strategies that rely on predictable individual responses and stable training context, to bring the highest upside, might fail when things do not roll-out as planned (and they never do) because these strategies do not protect from the downside. However, how does one protect from the downside?

One of the most researched robust strategy in asset allocation under uncertainty is $1 / N$ heuristic ${ }^{9,29-39}$, which simply allocates equally across all $\mathrm{N}$ funds under consideration. From a training perspective, this means that all five qualities receive equal training load. The archetypal $1 / \mathrm{N}$ loading strategy is microloading strategy, but what this strategy is lacking is higher load saturation into a single quality that might be needed to progress that quality forward in terms of adaptation $^{6-8}$. In the barbell chain loading strategy, the $1 / \mathrm{N}$ heuristic is utilized on the first day, and 
it is hypothesized that this strategy helps in increasing robustness of training when facing MCAR events.

Once protection from the downside is taken care off, one is free to invest in potentially risky, but highly rewarding funds. From training planning perspective this means allocating more training load into fewer qualities. This type of saturation is needed to yield adaptation, especially in highly trained individuals, which is the core idea behind block periodization ${ }^{6-8}$. An archetypal example of training load saturation to a single quality can be seen in concentrated loading strategy. In barbell loading strategy this constrained saturation is done by randomly emphasizing single quality in days two to five. This way, barbell strategy implements protection from the downside (by using $1 / \mathrm{N}$ heuristic on the day 1) and the pursuit of the upside (by randomly emphasizing certain quality). In the case of a missing session, barbell strategy aims to make sure all qualities receive an equal medium-high load to protect from the downside by restarting the chain on day one, which is hypothesized to increase robustness under MCAR conditions. Same restart principle is applied to ramp decreasing and block chain loading strategies (Figure 6 and 7).

\section{Simulation conditions}

Combining ten different loading strategies with three modification, total number of loading strategies utilized in simulation was 19: (1) concentrated, (2) concentrated random, (3) microloading, (4) hard-easy, (5) hard-easy random, (6) medium-easy, (7) medium-easy random, (8) increasing, (9) increasing random, (10) decreasing, (11) ramp decreasing, (12) ramp decreasing random, (13) ramp decreasing chain, (14) ramp increasing, (15) block, (16) block random, (17) block chain, (18) barbell random, and (19) barbell chain.

Each loading strategy is applied for 150 days, under normal conditions (no missing sessions), as well under MCAR conditions. The following MCAR conditions are being simulated: (1) no missing day (missing 0 in 10), (2) missing 1 in 10, (3) missing 2 in 10, (4) missing 4 in 10, and (5) missing 6 in 10 days. Each scenario for every loading strategy is simulated 100 times.

\section{Behavior metrics}

Training load for each quality, as well as for total training load (sum of training loads for all five qualities in a given day) is analyzed using exponential moving average (EMA) approach to get acute training load (rolling 7 days; alpha=0.25), and simple moving average (SMA) to get chronic training load (rolling 28 days), as well as acute to chronic workload ratio (ACWR) ${ }^{40-42}$ for every day in simulations.

Additional metric "between qualities standard deviation" (btwnQualitiesSD) is introduced to estimate variance in training load between qualities (using acute, chronic, and ACWR) (see Table 1). This is done to estimate unequal emphasis on qualities.

To estimate saturation of a single quality, ratio metric is utilized which represents the simple ratio between Quality01 (the first quality that needs to be developed) and total training load, using acute, 
chronic, and ACWR (Table 1). This is done to estimate how much emphasis Quality01 is receiving compared to the total training load.

\begin{tabular}{rrrrrrrrr} 
Day & Quality01 & Quality02 & Quality03 & Quality04 & Quality05 & Total & btwnQualitiesSD & Ratio \\
\hline 1 & 35.00 & 35.00 & 35.00 & 35.00 & 35.00 & 175.00 & 0.00 & 0.20 \\
2 & 28.75 & 28.75 & 28.75 & 36.56 & 28.75 & 151.56 & 3.49 & 0.19 \\
3 & 31.88 & 24.06 & 24.06 & 29.92 & 24.06 & 133.98 & 3.81 & 0.24 \\
4 & 26.41 & 20.55 & 20.55 & 32.75 & 20.55 & 120.80 & 5.43 & 0.22 \\
5 & 22.30 & 17.91 & 17.91 & 34.88 & 17.91 & 110.91 & 7.35 & 0.20 \\
6 & 25.48 & 22.18 & 22.18 & 34.91 & 22.18 & 126.93 & 5.51 & 0.20 \\
7 & 21.61 & 19.14 & 19.14 & 28.68 & 26.95 & 115.51 & 4.46 & 0.19 \\
8 & 18.71 & 24.67 & 16.85 & 24.01 & 22.71 & 106.95 & 3.43 & 0.17 \\
9 & 16.53 & 21.00 & 15.14 & 20.51 & 27.35 & 100.52 & 4.77 & 0.16 \\
10 & 14.90 & 18.25 & 21.67 & 17.88 & 23.01 & 95.70 & 3.23 & 0.16 \\
11 & 19.92 & 22.44 & 25.00 & 22.16 & 26.01 & 115.53 & 2.42 & 0.17 \\
12 & 25.25 & 19.33 & 21.25 & 19.12 & 22.01 & 106.96 & 2.49 & 0.24 \\
13 & 21.44 & 24.81 & 18.44 & 16.84 & 19.00 & 100.53 & 3.10 & 0.21 \\
14 & 18.58 & 21.11 & 16.33 & 22.94 & 16.75 & 95.71 & 2.84 & 0.19 \\
15 & 24.25 & 18.33 & 14.75 & 19.71 & 15.06 & 92.10 & 3.89 & 0.26 \\
16 & 26.94 & 22.50 & 19.81 & 23.53 & 20.05 & 112.82 & 2.91 & 0.24 \\
17 & 30.51 & 19.37 & 17.36 & 20.15 & 17.54 & 104.93 & 5.46 & 0.29
\end{tabular}

Table 1. Calculation of betweenQualitiesSD and Ratio metrics for Acute loading using Qualities 1 to 5.

Behavior metrics include (1) acute, chronic and ACWR for all five qualities and total training load, (2) btwnQualitiesSD for acute, chronic and ACWR, and (3) ratio between Quality01 and total training load for acute, chronic and ACWR. Since the behavior metrics are calculated for every day, every loading strategy, every type of MCAR condition, and every simulation, it represents a tremendous amount of data to be visualized and statistically analyzed (see Supplementary Materials for extensive visual analysis). To represent this data in a more compact and visually appealing way, simple descriptive statistics of mean, minimum and maximum were utilized across all days and all simulations for every loading strategy and MCAR conditions.

Additional analysis using total sum and SD of daily loads over 150 days for all qualities and total training load was performed and summarized using minimum, mean and maximum across all simulations for every loading strategy and MCAR conditions.

Using summary statistics of the behavior metrics, hierarchical clustering using Ward's method ${ }^{43,44}$ was performed to help visualize and find loading strategies with similar behavior, as well as to sort out labels on graphs for easier visual pattern recognition. Reproducible R code, for both simulation and analysis, is available in the Supplementary Materials. 


\section{Results}

Single simulation visuals and performance

Visualization of a single simulation without missing sessions can be seen in figures 9-12. More specifically, Figure 9 depicts acute EMA and chronic SMA for Quality01 whereas Figure 10 depicts acute and chronic exponential rolling averages for total training load. Furthermore, Figure 11 shows btwnQualitiesSD for acute and chronic metrics while Figure 12 illustrates the ratio between Quality01 and total training load for acute and chronic metrics.
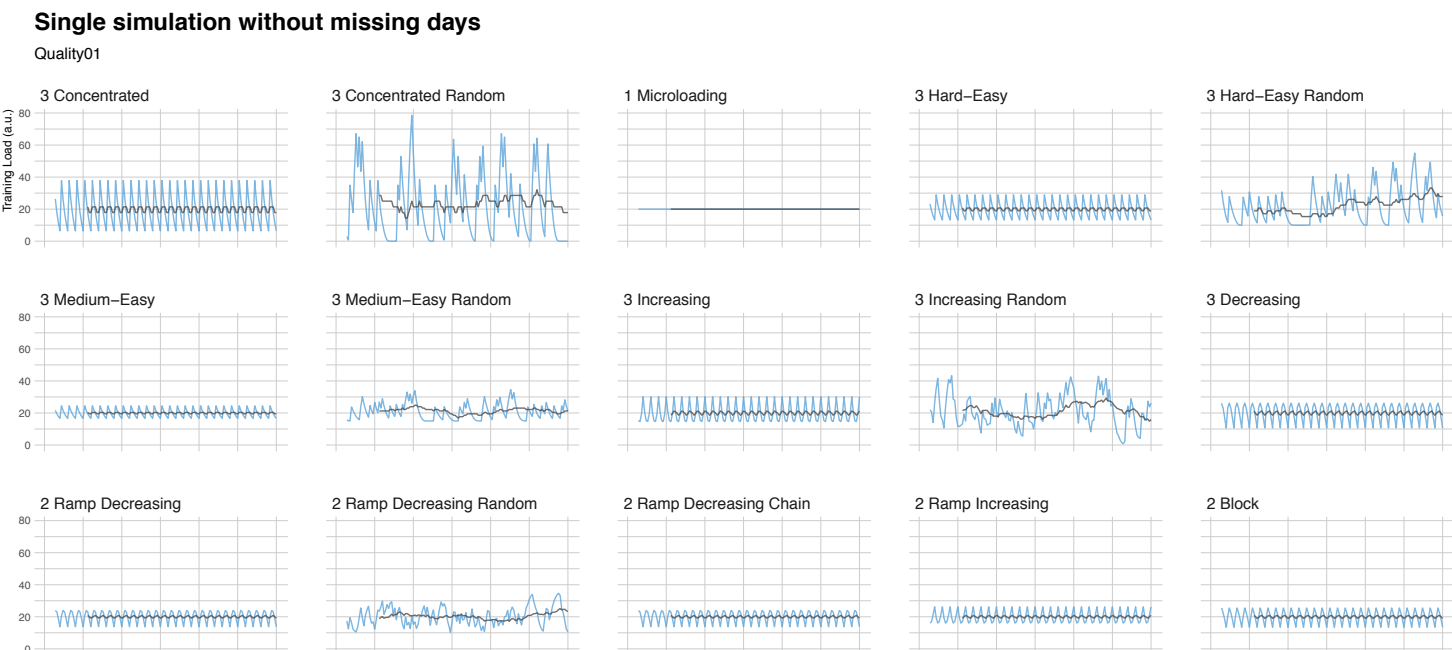

2 Ramp Increasing
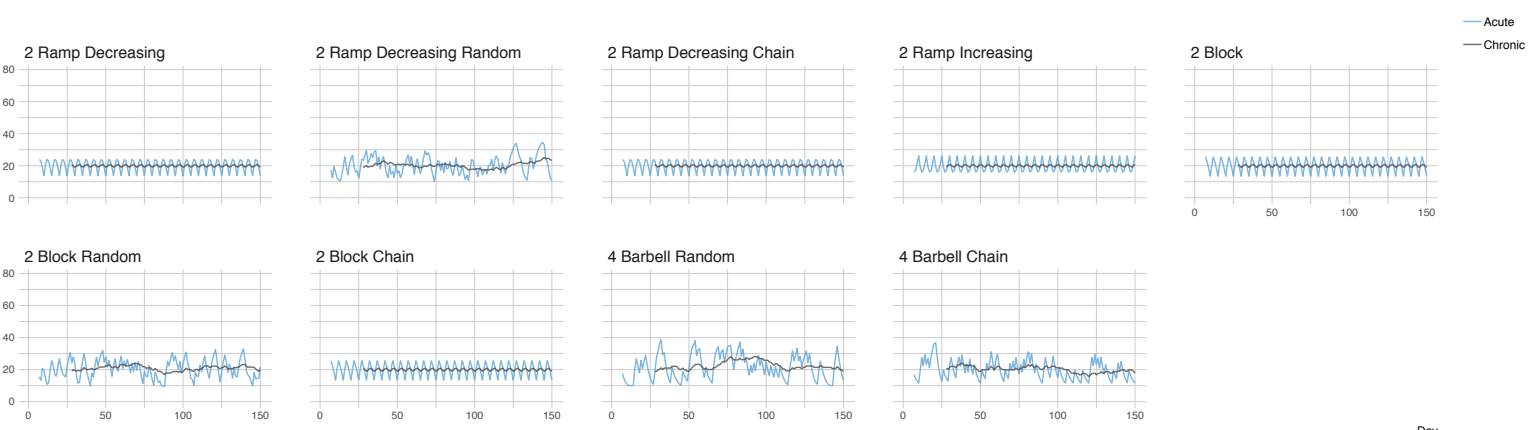

Figure 9. Acute and chronic training load for Quality01 over 150 days 


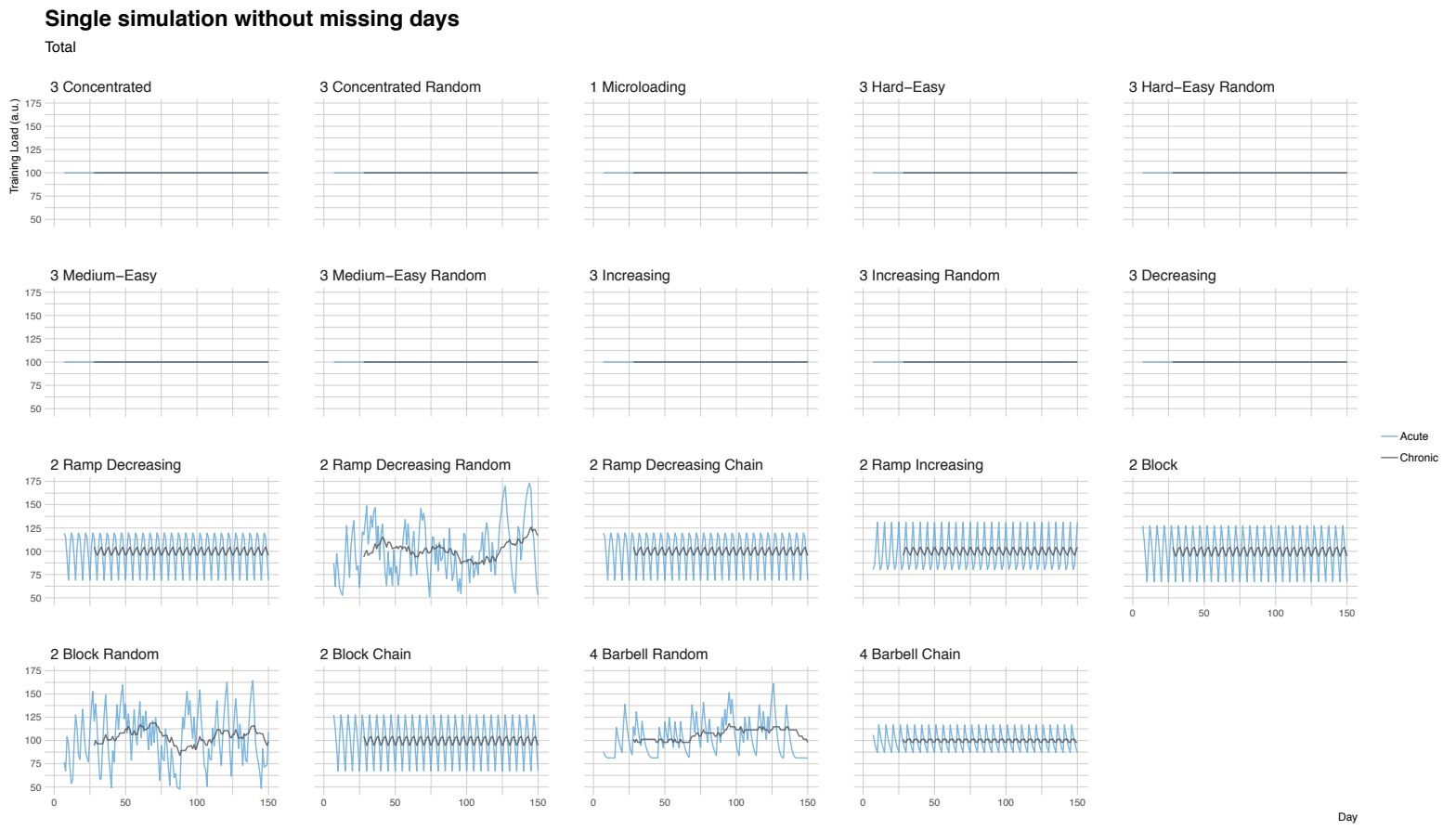

Figure 10. Acute and chronic training load for total load over 150 days
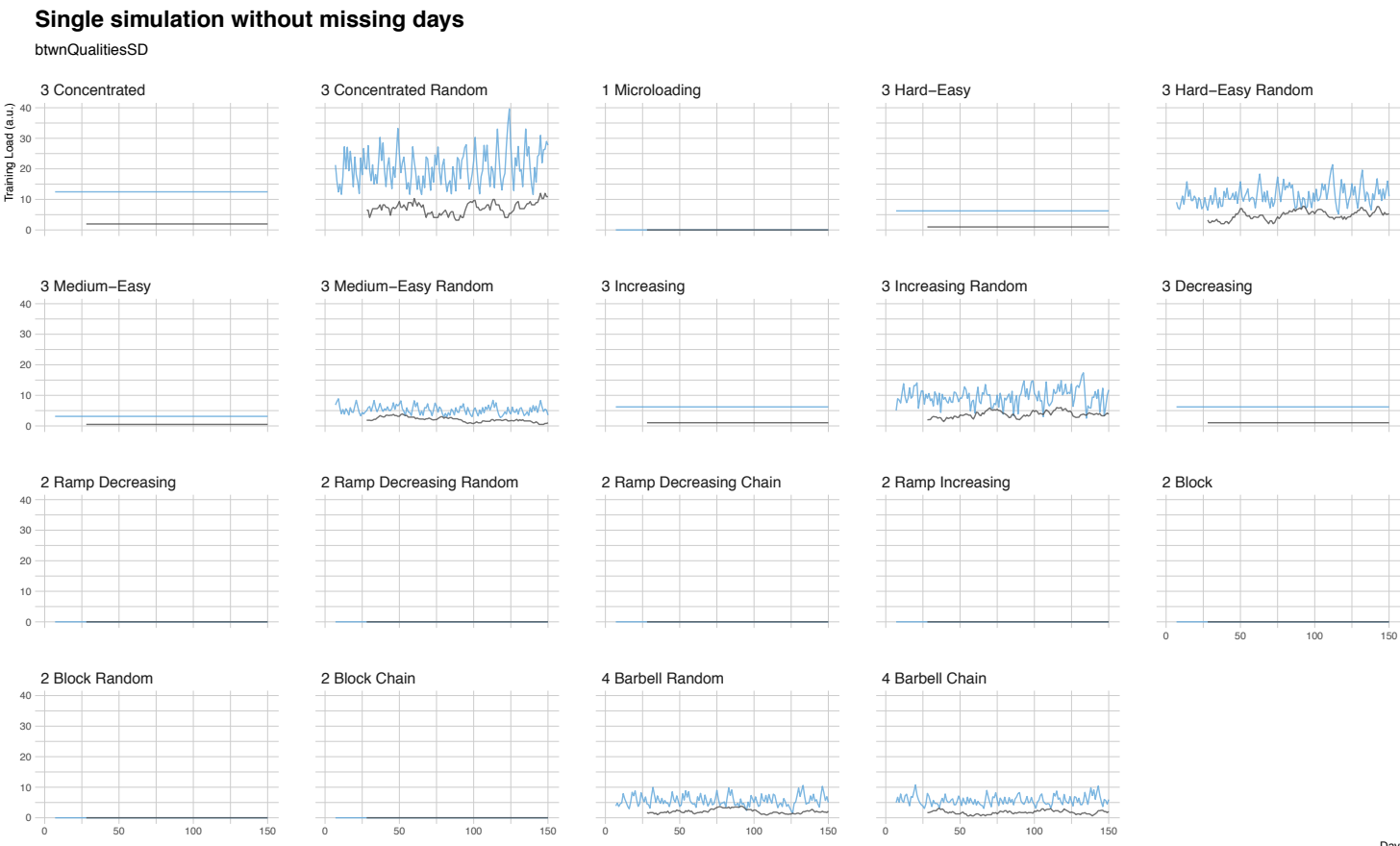

Figure 11. Between qualities SD for acute and chronic training loads over 150 days 


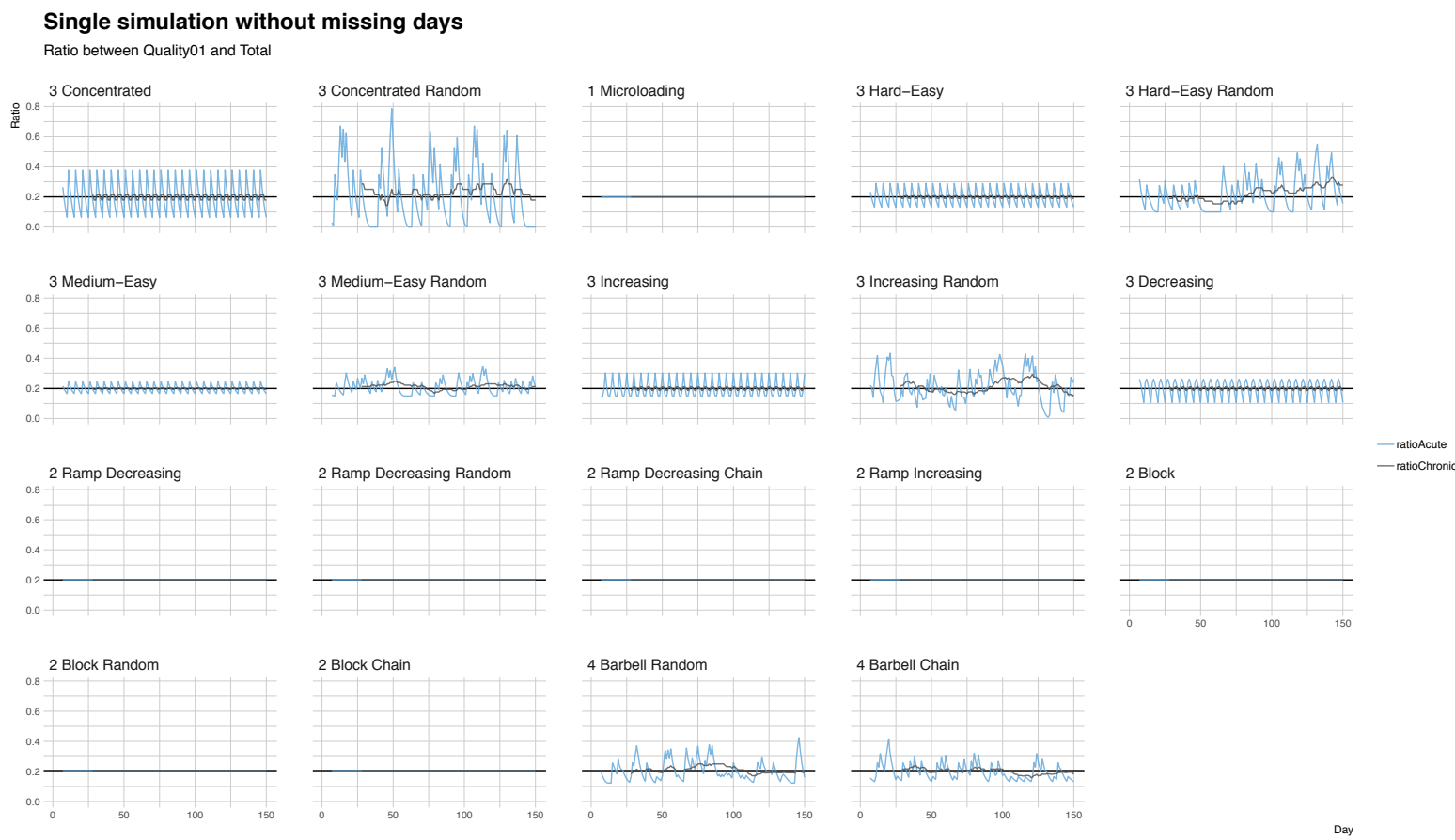

Figure 12. The Ratio between Quality01 and total load acute and chronic training loads over 150 days. The horizontal black line represents a theoretical $20 \%$ distribution (total load per day is 100, and there are 5 qualities).

Due to missing data in the first 28 days (as it takes to estimate chronic training load), this time frame is removed in the analysis involving acute, chronic and ACWR metrics.

The total sum of daily loads

The total sum of daily loads for all five qualities and total training load in MCAR condition over 150 days can be seen in Figure 13 while hierarchical clustering of the summaries for MCAR conditions using descriptive statistics is presented in Figure 14. Hierarchical clustering is used to explore and visualize similarities in loading strategies behavior. 


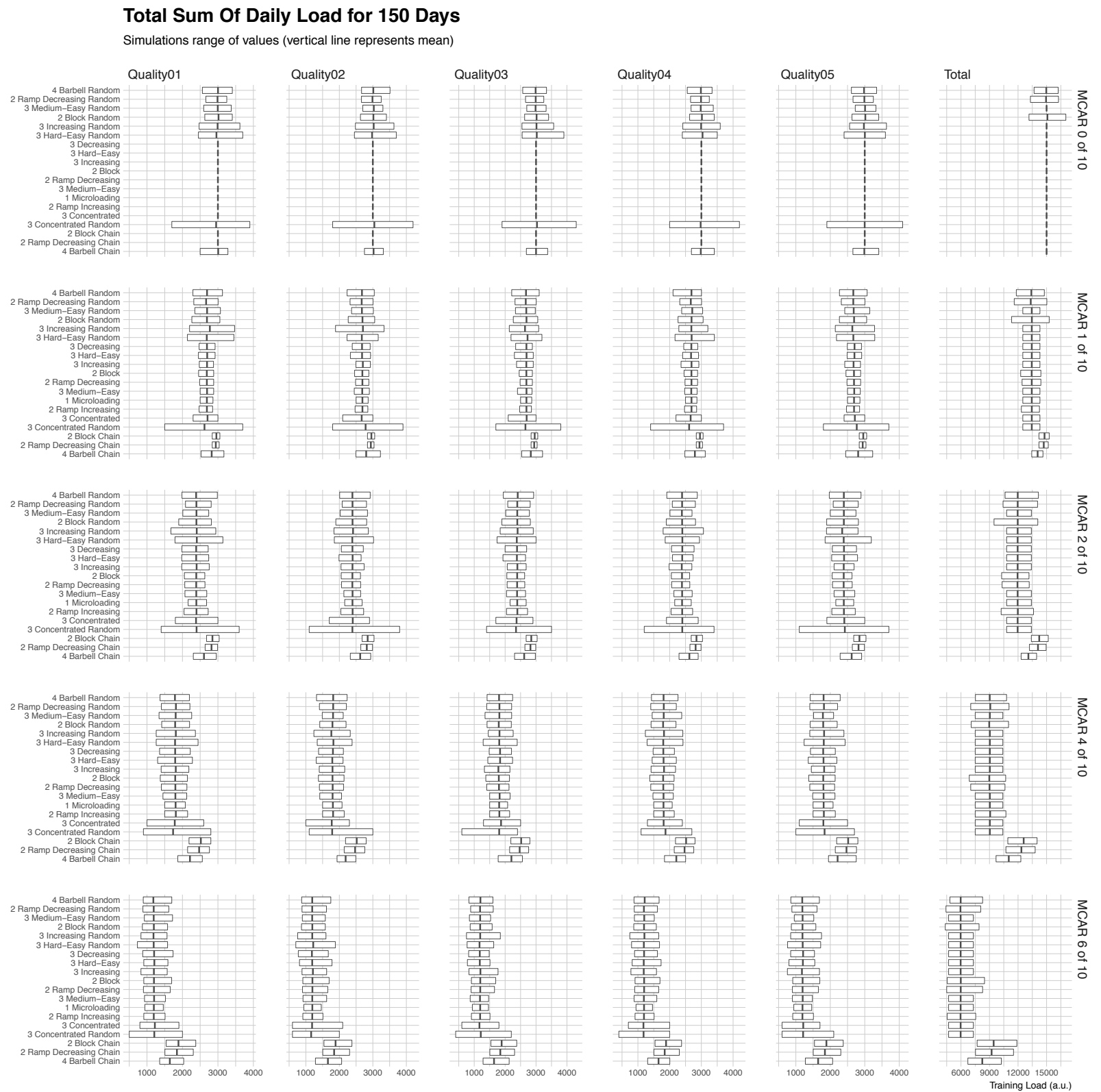

Figure 13. The total sum of training loads per qualities and total training load over 150 days for different MCAR conditions. Squares represent minimum and maximum, and vertical line represents mean in 100 simulations. Loading strategies are sorted based on hierarchical clustering. Numbers before loading strategy refer to a belonging quadrant. 


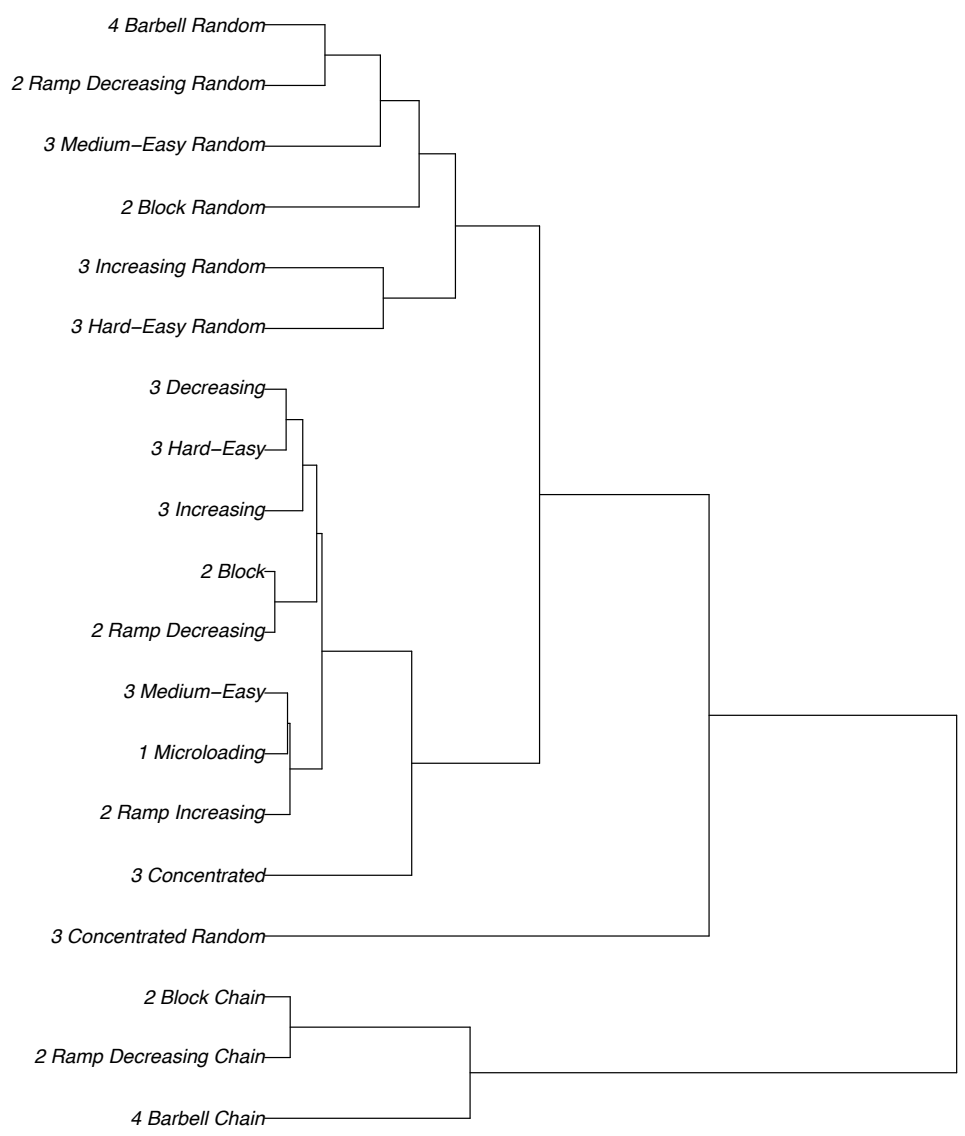

Figure 14. Hierarchical clustering using Ward's method for MCAR conditions utilizing minimum, mean and maximum of the total sum of training loads per qualities and the total training load over 150 days for all simulations. Numbers before loading strategy refers to a belonging quadrant

From these figures, it can be seen that the chain loading strategies differentiate themselves from other loading strategies in maintaining both the total training load, as well as training load directed towards qualities in the face of MCAR conditions. Moreover, random variations of loading strategies seem to be forming another cluster, but the reason for that is probably higher variation in training load, particularly in no missing days events (MCAR 0 of 10). This seems to be the reason for other loading strategies to form a cluster, although concentrated random loading strategy seems to be an outlier. In summary, there seem to be four clusters of loading strategies when it comes to the total sum of training load over 150 days in the face of MCAR conditions. Additional cluster stability and the optimal number of cluster analysis were not performed.

\section{The standard deviation of daily loads}

Figure 15 shows SD of daily loads for all five qualities and total training load in MCAR condition over 150 days whereas hierarchical clustering of the summaries for MCAR conditions using descriptive statistics can be seen in Figure 16. 


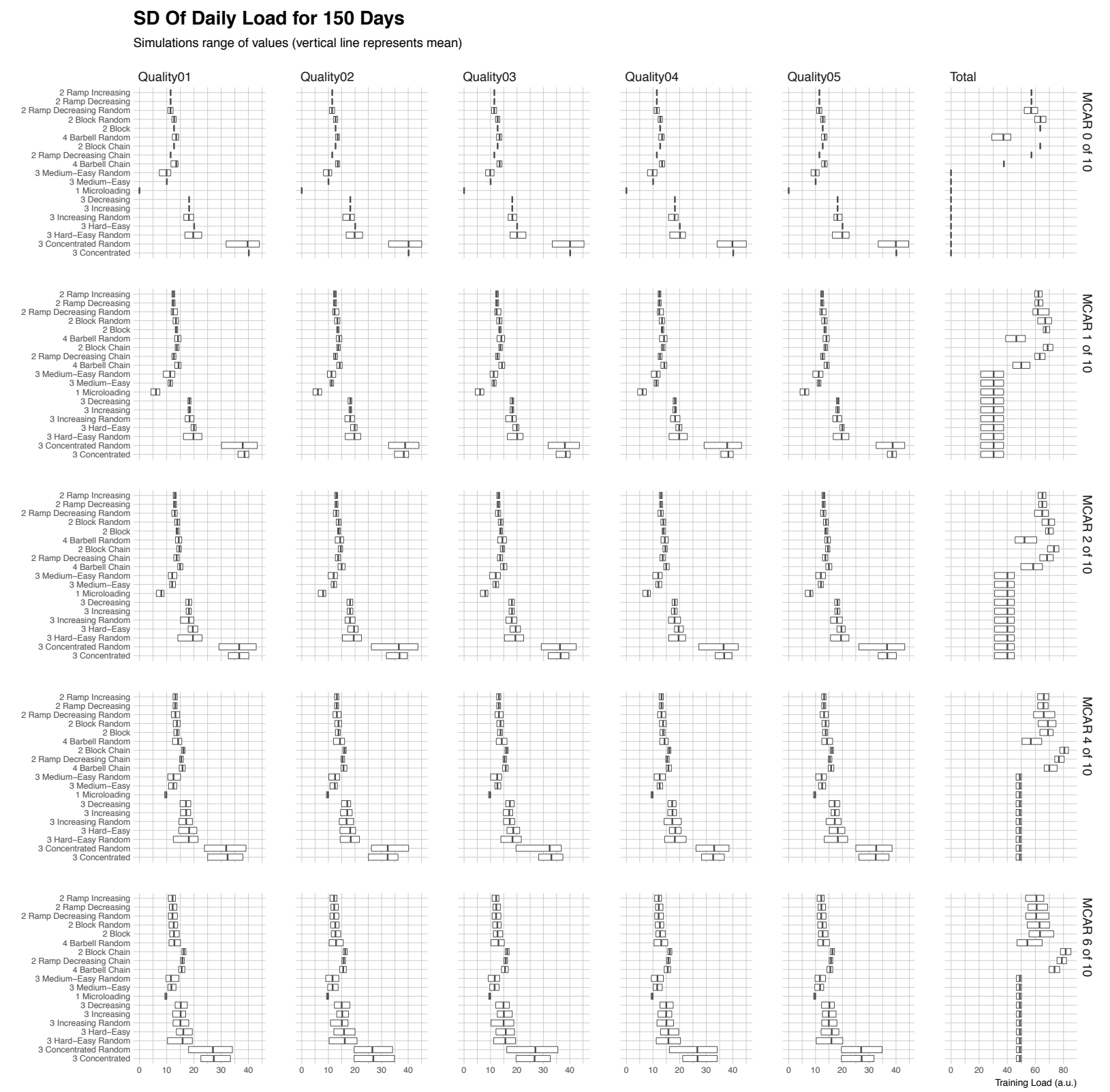

Figure 15. Training load standard deviation per qualities and total training load over 150 days for different MCAR conditions. Squares represent minimum and maximum, and vertical line represents mean in 100 simulations. Loading strategies are sorted based on hierarchical clustering. Numbers before loading strategy refers to a belonging quadrant. 


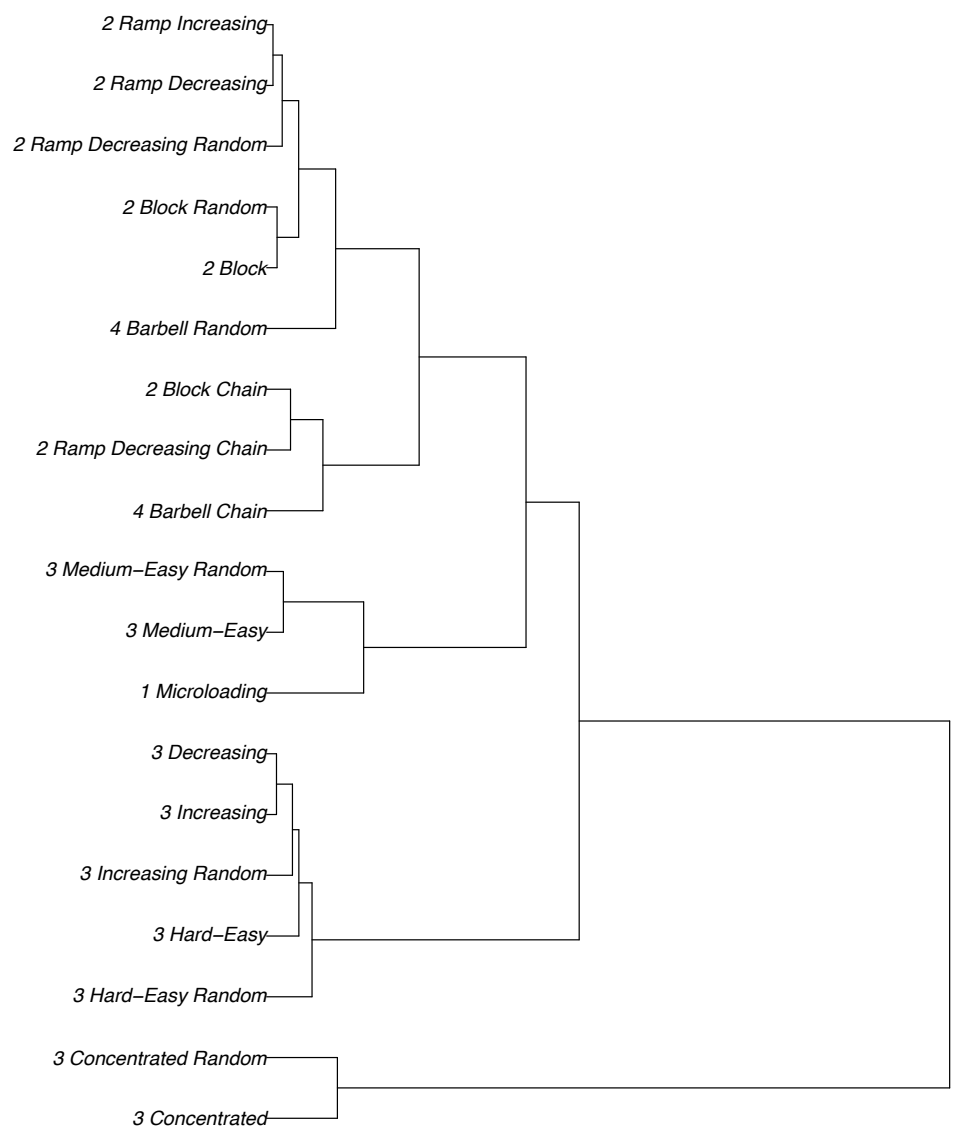

Figure 16. Hierarchical clustering using Ward's method for MCAR conditions utilizing minimum, mean and maximum of the standard deviation of training loads per qualities and total training load over 150 days for all simulations. Numbers before loading strategy refers to a belonging quadrant

Results of the simulation presented in figures 15 and 16 suggest that concentrated and concentrated random loading strategies are forming a separate cluster due to higher SD of daily training loads. Non-flat loading strategies (quadrants 2 and 4) seems to be forming one cluster, with chain loading strategies as a sub-cluster. Loading strategies from quadrants 1 and 3 (flat loading) seem to be forming a cluster of similar behavior in MCAR events, with medium-easy, medium-easy random and microloading strategies forming additional cluster due to their lower SD in qualities training load. Additional cluster stability and the optimal number of cluster analysis were not performed.

\section{Acute and chronic load, and ACWR}

Summary of the simulation data, using descriptive statistics for acute and chronic load and ACWR for Quality01, total load, btwnQualitieSD, and the ratio of Quality01 to total load under MCAR conditions is presented in figures 17-20. 


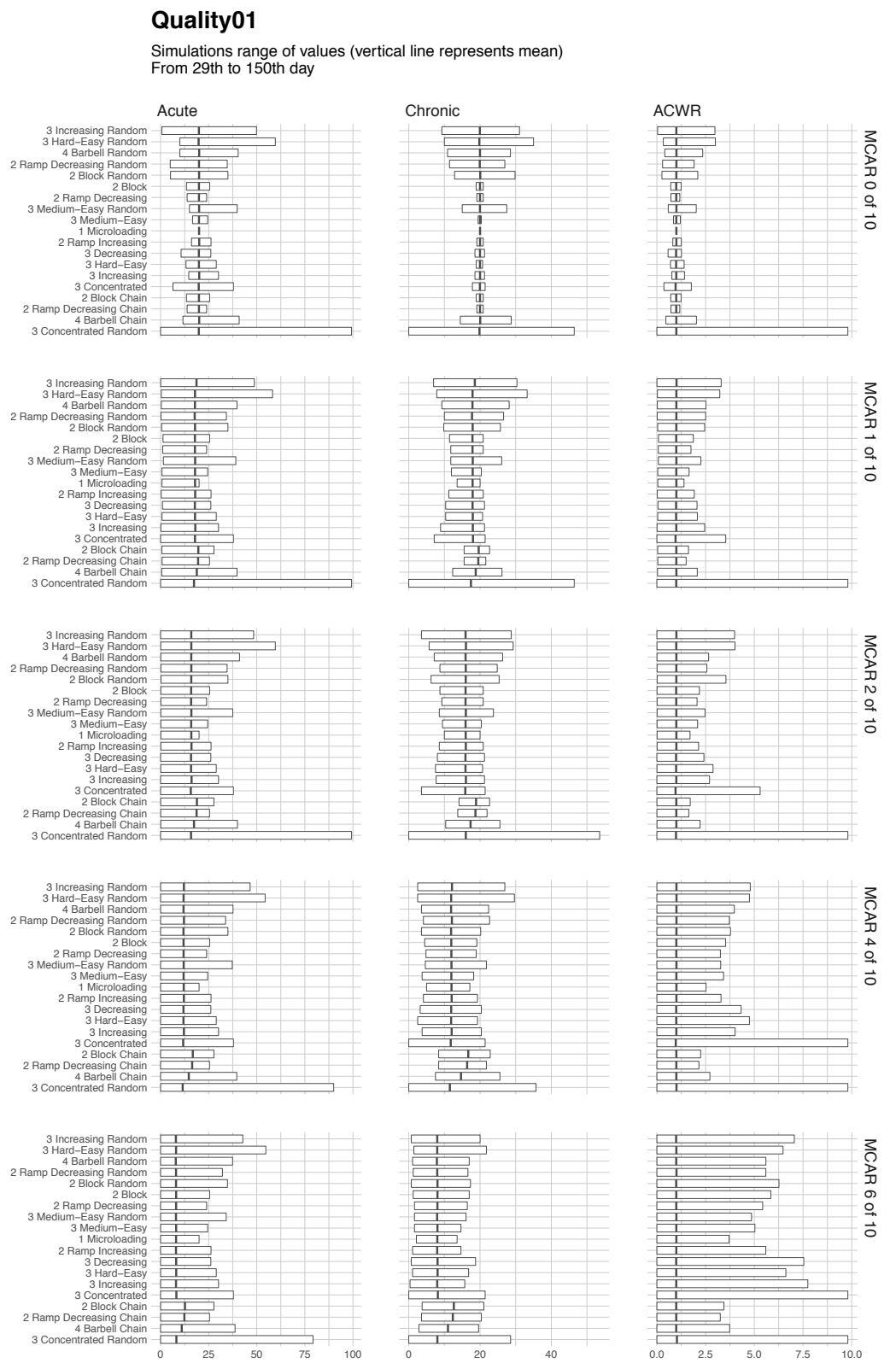

Figure 17. Simulation range of values for acute and chronic load, and ACWR across different MCAR conditions for Quality01. Loading strategies are sorted based on hierarchical clustering. Numbers before loading strategy refer to a belonging quadrant

More specifically, Figure 17 depicts simulation ranges for acute and chronic load, and ACWR of Quality01 across different MCAR conditions for loading strategies (see Supplementary materials for more visuals). Concentrated and concentrated random loading strategies seems to represent two outliers (particularly concentrated random) and they tend to form two separate clusters. Chain strategies are forming separate cluster themselves, mostly because they show higher mean across simulations in acute and chronic metrics. Random variants of loading strategies (except mediumeasy random) seems to be forming one cluster, mainly due to higher SD of quality loading, particularly in no MCAR conditions. All other loading strategies seem to be forming one large 
cluster. Visual inspection indicates that there seem to be five clusters, although cluster stability and the optimal number of cluster analysis were not performed.

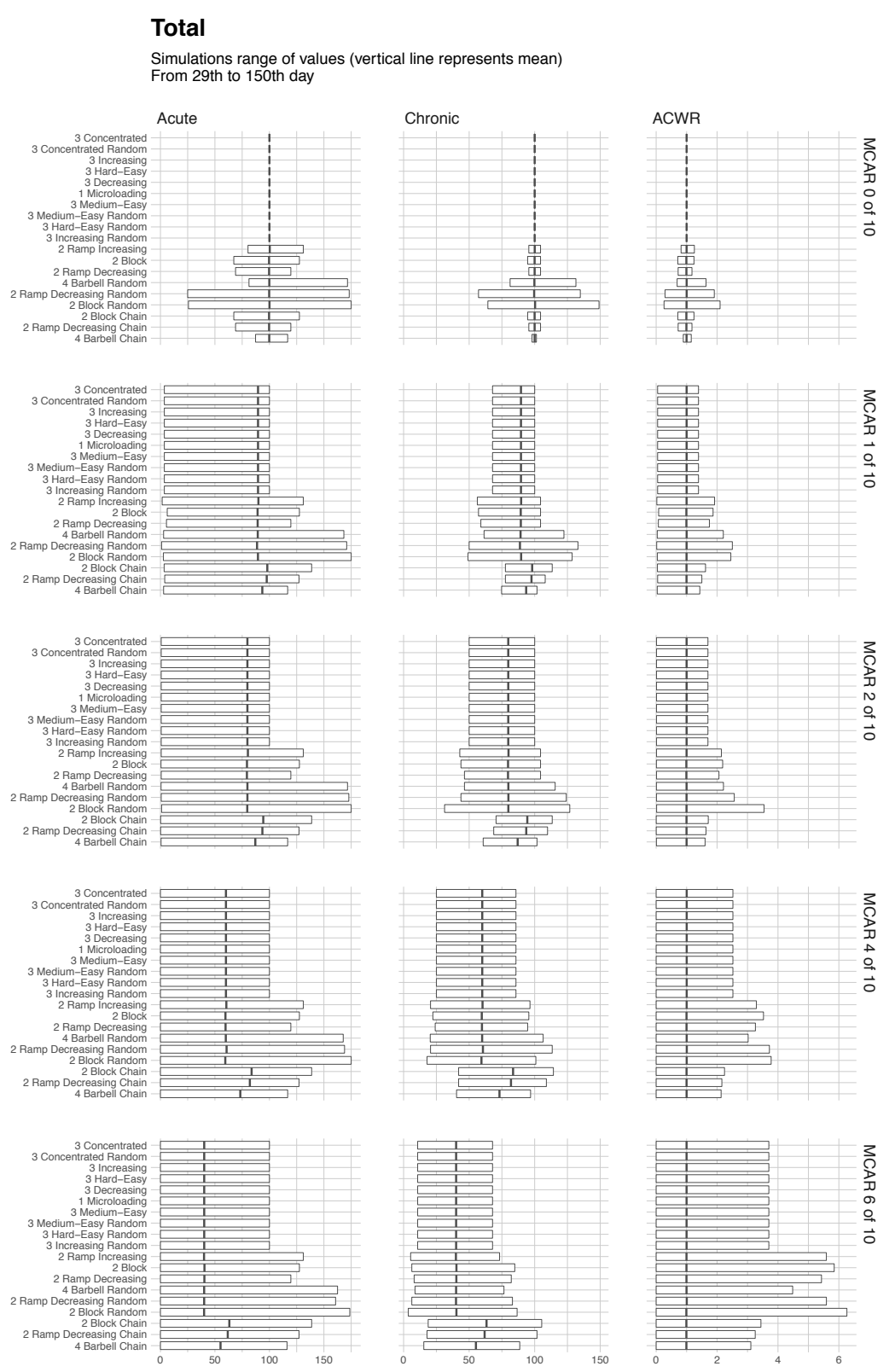

Figure 18. Simulation range of values for acute and chronic load, and ACWR across different MCAR conditions for total load. Loading strategies are sorted based on hierarchical clustering. Numbers before loading strategy refers to a belonging quadrant

Furthermore, when it comes to total load (Figure 18), it can be seen that flat loading strategies (quadrants 1 and 3 ) are making a separate cluster due their same behavior across MCAR conditions (see Supplementary materials for more visuals). Chain strategies are forming one cluster, and their random variants are forming one cluster as well. Chain strategies are showing lower variability, and higher mean in acute and chronic metrics across different MCAR conditions. Visual inspection 
indicates that there seem to be three or four clusters, although cluster stability and the optimal number of cluster analysis were not performed.

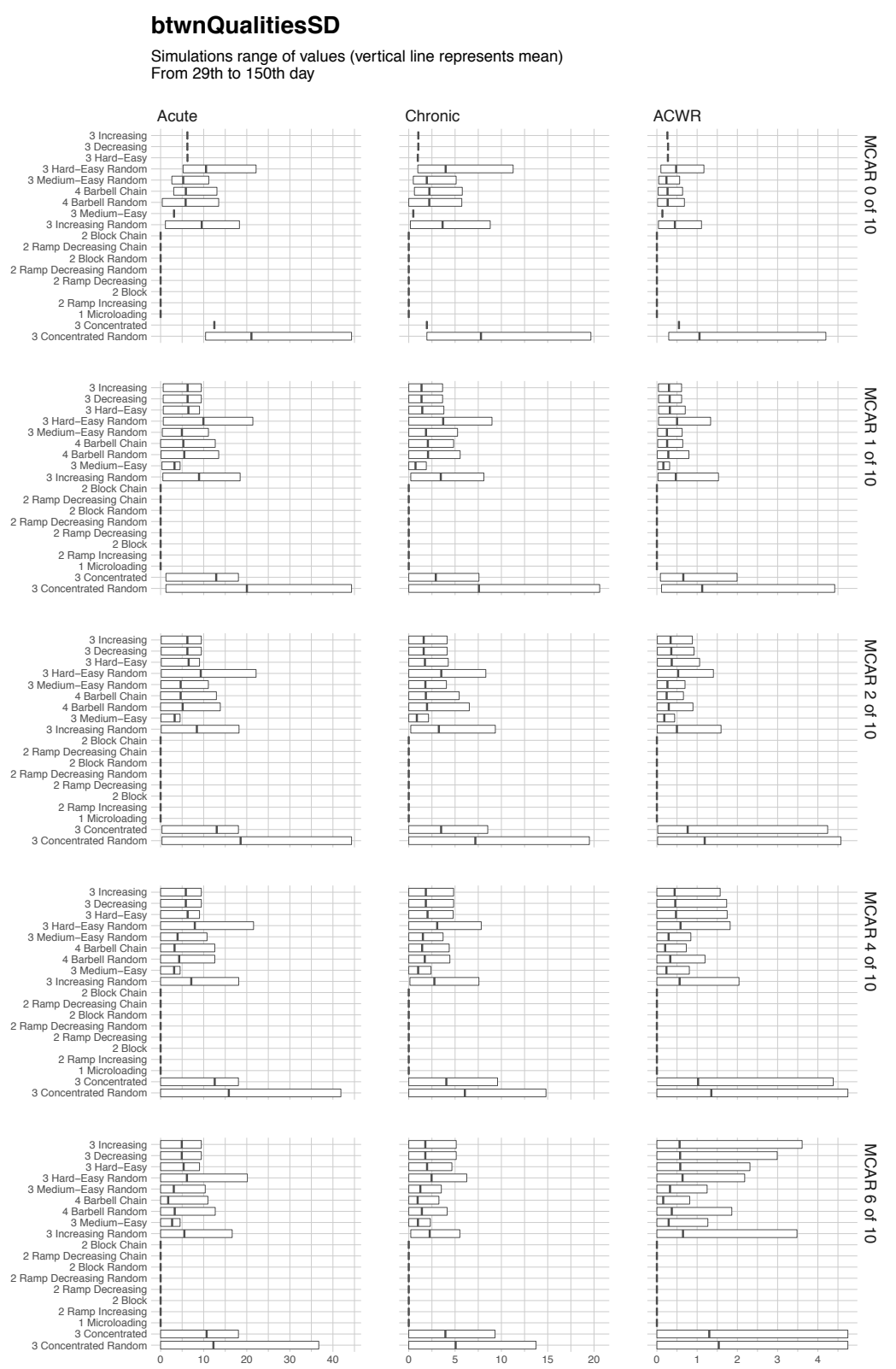

Figure 19. Simulation range of values for acute and chronic load, and ACWR across different MCAR conditions for between qualities SD. Loading strategies are sorted based on hierarchical clustering. Numbers before loading strategy refers to a belonging quadrant

When analyzing between qualities SD (Figure 19), loading strategies that have equal training load distribution per qualities (quadrants 1 and 2) per day seems to be forming a separate cluster for between qualities SD, which is zero in all cases (see Supplementary materials for more visuals). This means that on every day in every simulation acute and chronic load, and ACWR between qualities are equal. Concentrated and concentrated random loading strategies seem to be forming a separate cluster. The rest of loading strategies forming a cluster are loading strategies with un- 
equal training distribution of quality per day (quadrants 3 and 4). Visual inspection indicates that there seem to be three clusters, although cluster stability and the optimal number of cluster analysis were not performed.

Figures 20 depicts results (minimum, mean, and maximum) of simulations using Quality01 and total load ratio between acute and chronic load, and ACW under different MCAR conditions.

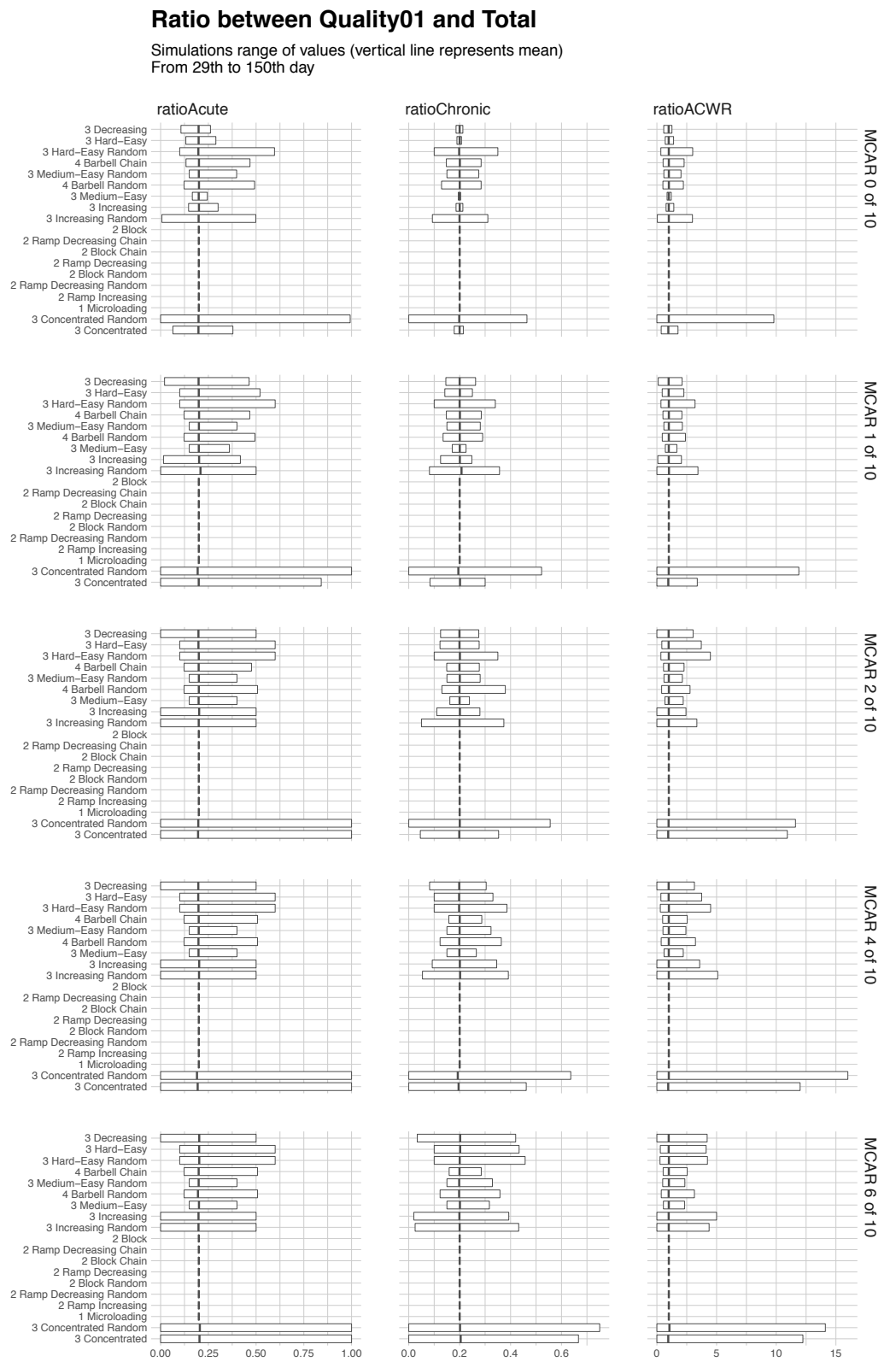

Figure 20. Simulation range of value of the ratio between Quality01 and total training load using acute and chronic load, and ACWR across different MCAR conditions. Loading strategies are sorted based on hierarchical clustering. Numbers before loading strategy refers to a belonging quadrant 
Equally distributed loading strategies (quadrant 1 and 2) seems to be forming one cluster due to a stable ratio ( 0.2 for acute and chronic, and 1.0 for ACWR) across simulations under MCAR conditions (see Supplementary materials for more visuals). Concentrated and concentrated random loading strategies seems to be forming a separate cluster, with the rest of loading strategies forming an additional cluster. Visual inspection indicates that there seem to be three clusters, although cluster stability and the optimal number of cluster analysis were not performed.

\section{Overall cluster analysis}

Merging all descriptive metrics into a single table result in 360 total features explaining the behavior of loading strategies in simulation under MCAR conditions. Performing hierarchical clustering results in the following clusters (Figure 21).

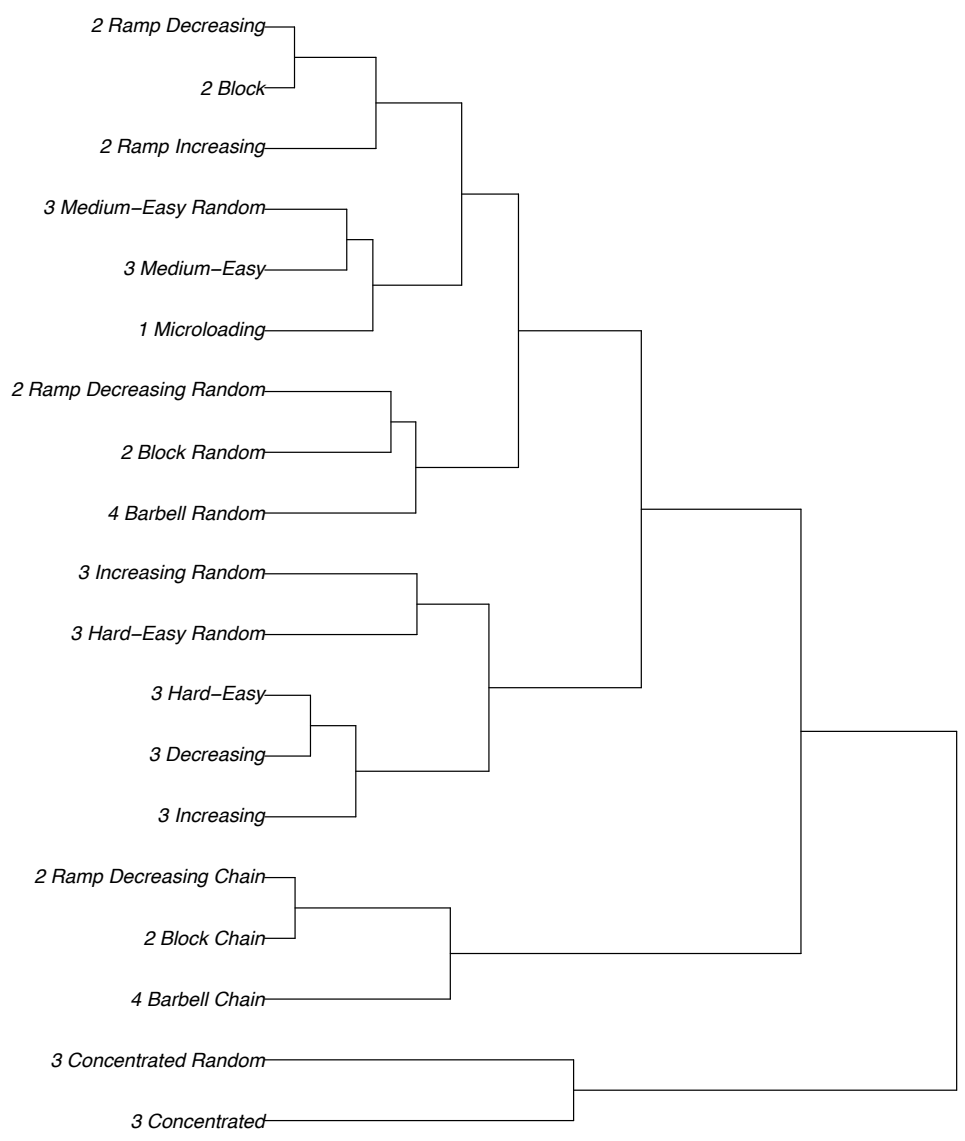

Figure 21. Hierarchical clustering using Ward's method using all descriptive statistics from the results of MCAR conditions simulations. Numbers before loading strategy refers to a belonging quadrant

Visual inspection indicates that there seem to be three to four clusters of behavior, although cluster stability and the optimal number of cluster analysis were not performed. Concentrated and concentrated random loading strategies seem to be forming one cluster in the overall behavior under MCAR conditions. Chain loading strategies seem to be forming a separate cluster. Unequally distributed loading strategies (quadrant 3) with a higher SD of quality training load distribution seems to be forming another separate cluster (hard-easy, decreasing, increasing, and their random 
variants). Non-flat loading strategies (quadrants 2 and 4), together with the rest of flat-loading strategies (microloading, medium-easy, and medium-easy random) appears to be forming their separate cluster.

\section{Discussion}

The significant insight from the simulations performed in the current study is the maintenance of the total training load, as well as loads directed at individual qualities using chain loading strategies, as hypothesized in the introduction. This characteristic makes chain loading strategies more robust when facing MCAR conditions. The predicted fragility of concentrated loading strategies (both normal and random variants) under MCAR conditions was also borne out. Concentrated loading strategies represent archetypal loading strategy where one puts 'all their eggs in one basket' - or, in other words, distributes the whole training load points to one quality on a given day, which might represent optimal sequence for developing qualities. This approach might be optimal under predictable conditions, but fragile under MCAR conditions, where robust strategies (e.g., chain, random) might not be optimal under predictable conditions, but perform better than optimal strategies under MCAR conditions. This fragility can be even more problematic in MNAR conditions, where one might miss a particular day(s) (e.g., missing day 2).

The archetypal example of $1 / \mathrm{N}$ heuristic is the microloading strategy, which, while it is the most robust loading strategy, suffers from reduced overall training load when facing MCAR events. Besides, we are speculating that this approach might fail to induce adaptation due lower and equally distributed training loads aimed at qualities 1 to 5 (which might be the case with all equally distributed loading strategies (quadrants 1 and 2) ${ }^{6-8}$. This is similar to medium-easy strategies, which microloading often clusters with. The more equally distributed loading strategy is (i.e., the more it approaches $1 / \mathrm{N}$ heuristic) the more robust it is, but less saturation might provide fewer stimuli for a given quality and thus lower adaptation and improvement. On the flip side, the more saturation (the more unequally distributed loading strategy) there is, the more emphasis a particular quality gets, the more fragile the strategy under MCAR and MNAR events. The reconciliation between these two strategies ( $1 / \mathrm{N}$ and saturating given quality) is theoretically done with the barbell chain loading strategy.

In the case of MNAR conditions, which are not simulated in the current paper, we speculate that random variations of loading strategies help in avoiding systematic (or planned) missed sessions. This way, randomness helps in increasing robustness in the face of MNAR conditions. Speculatively, an additional benefit of randomization of loading strategies might involve making athletes more adaptable to changing training loads aimed at different qualities, rather than adapting to a particular sequence. On the flip side, this represents a potential downside due to residual effects and interactions between qualities development. For example, developing Quality01 on Day 1 might interfere with developing Quality02 on Day 2 due to residual fatigue or interference effects. The question then becomes how to reconcile randomization with apriori knowledge about the appropriate sequence? One potential solution might be Markov models ${ }^{45}$, where transition (and 
thus sequence) from a particular day (or emphasis) can be probabilistically expressed and modeled. Using the Markov model, one can embed apriori knowledge about the appropriate sequence of training loads (e.g., hard, medium, easy) and aims (e.g.. Quality01, Quality02).

Markov models are represented either with a network or with a transition matrix. Although the name sounds a bit complex, Markov models are quite simple, and we are already using them. For example, Figure 22 depicts Markov model transition matrix for concentrated, concentrated random and chain loading strategies.

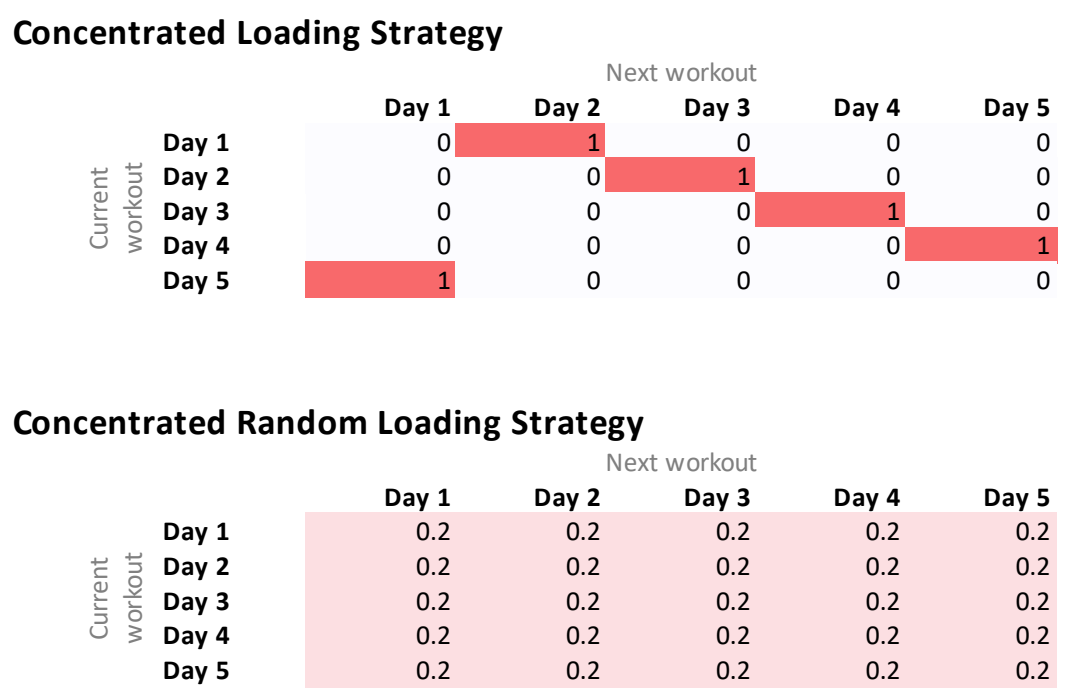

Chain Loading Strategies

\begin{tabular}{|c|c|c|c|c|c|c|c|}
\hline & & & & Next $w$ & & & \\
\hline & & Missing Day & Day 1 & Day 2 & Day 3 & Day 4 & Day 5 \\
\hline & Missing Day & 0 & 1 & 0 & 0 & 0 & 0 \\
\hline & Day 1 & 0 & 0 & 1 & 0 & 0 & 0 \\
\hline c) & Day 2 & 0 & 0 & 0 & 1 & 0 & 0 \\
\hline 둔 & Day 3 & 0 & 0 & 0 & 0 & 1 & 0 \\
\hline & Day 4 & 0 & 0 & 0 & 0 & 0 & 1 \\
\hline & Day 5 & 0 & 1 & 0 & 0 & 0 & 0 \\
\hline
\end{tabular}

Figure 22. Markov model transition matrix for concentrated, concentrated random, and chain loading strategies. Numbers in the tables represent the probability of transition from the current workout to the next. The rows must sum to one.

Taking concentrated loading strategy as an example, the Markov model transition table defines a non-random sequence from Day 1 to Day 2, from Day 2 to Day 3 and so forth. The random variant of concentrated loading strategy has a uniform probability of 0.2 across the transition matrix, which means that from any current day, every other day has equal chance to be selected as the next in sequence. Chain loading strategies have an additional state in the transition matrix, which is a missing day. In chain strategies, the current missing day always leads to Day 1 (expressed as a probability of 1). This way, every time there is a missing day, the chain is restarted. In the barbell chain loading strategy, this first day represents the most laborious day with $1 / \mathrm{N}$ heuristic (all five qualities receive equal training load points), while the other days represent a random emphasis on 
qualities. This uniform randomness from Day 1 to Day 2 in barbell chain can be improved by combining an apriori known sequence of qualities development, by giving a higher probability to the appropriate sequence (while still allowing random sequence). Figure 23 illustrates an example of an improved barbell chain by implementing higher chance for following a given sequence.

\begin{tabular}{|c|c|c|c|c|c|c|c|c|}
\hline & \multicolumn{8}{|c|}{ Next Workout } \\
\hline & Missing Day & $1 / \mathrm{N}$ & Quality 1 & Quality 2 & Quality 3 & Quality 4 & Quality 5 & Day Off \\
\hline Missing Day & 0 & 1 & 0 & 0 & 0 & 0 & 0 & 0 \\
\hline $1 / N$ & 0 & 0.1 & 0.5 & 0.1 & 0.1 & 0.1 & 0.1 & 0 \\
\hline Quality 1 & 0 & 0 & 0 & 0.5 & 0.3 & 0.1 & 0.1 & 0 \\
\hline Quality 2 & 0 & 0 & 0 & 0 & 0.5 & 0.3 & 0.2 & 0 \\
\hline Quality 3 & 0 & 0 & 0.1 & 0.1 & 0 & 0.5 & 0.3 & 0 \\
\hline Quality 4 & 0 & 0 & 0.1 & 0.1 & 0.1 & 0.1 & 0.5 & 0.1 \\
\hline Quality 5 & 0 & 0 & 0 & 0 & 0 & 0 & 0 & 1 \\
\hline Day Off & 0 & 0.4 & 0.6 & 0 & 0 & 0 & 0 & 0 \\
\hline
\end{tabular}

Figure 23. Markov model transition matrix for improved barbell chain loading strategy. Numbers in the tables represent the probability of transition from the current workout to the next. The rows must sum to one.

The improved barbell chain loading (Figure 23) implements an apriori known sequence of quality development. The sequence might start at $1 / \mathrm{N}$ day or Quality 1 day. In the case of the missing day, the chain restart with the highest probability $(0.5)$ at $1 / \mathrm{N}$ day, although it can restart with any other days as well (except Day Off). Once the 1/N day is done, emphasis on Quality01 receives the highest probability. This sequence can be based on apriori knowledge of appropriate qualities development sequencing, but it can also involve known individual needs of a given individual (or a team) where certain qualities might demand higher likelihood of emphasis. For example, a given individual might need more resistance training (e.g., Quality01), thus this quality (or a method) might be represented with higher chances in the transition matrix. This way one can combine an appropriate sequence of the workouts, individual needs (which represent optimal approach) while using a bit of randomness to make athlete adaptable while using barbell chain to make sure planning is robust in the face of MCAR and MNAR events.

Besides embedding the apriori sequence of quality emphasis, the Markov model can be used to embed the loading sequence (e.g., hard, medium, easy), and this can represent an additional transition matrix. The probabilities in this transition matrix can be dynamic or in other words based on readiness monitoring ${ }^{40}$, which might include wellness monitoring (e.g., subjective ratings or heart rate variability) or performance monitoring (e.g., jump testing). For example, if a particular athlete shows worse than normal ratings, then the training load transition matrix can be updated so that easy training load (or day off) receives a higher chance. Using multi-layered Markov models, one can integrate barbell chain strategy (and improve robustness), appropriate sequencing of the workouts (or quality emphasis), individual needs, and individual readiness to train. To our knowledge, this is the first time such an approach is suggested. It was beyond the scope of the current paper to investigate this approach to planning, although in our opinion, fruitful probabilistic approach to planning.

These approaches are not without drawbacks. Before jumping to hard $1 / \mathrm{N}$ day, one might want to know why a given athlete keeps missing specific sessions, or sessions overall. Could it be it is dues 
boredom, injury, over-reaching or something else? If someone misses a longer sequence of a training session, is the hardest day using $1 / \mathrm{N}$ heuristic the most appropriate approach to reintroducing athletes to training? Using the Markov model, maybe this long sequence of missing sessions can be modeled as a specific state, that results in a training restart with an easier session utilizing $1 / \mathrm{N}$ heuristic. This way, someone missing a longer sequence of workouts will be reintroduced to training with less than a hard-load $1 / \mathrm{N}$ type of workout. In any case, one can probably introduce different layers of Markov models, for different time frames (e.g., break-in phase might be characterized by easy-medium load and microloading strategies, while peaking phase might be more of a saturation toward key qualities over multiple weeks). To our knowledge, this represents a novel approach to periodization, that could easily be implemented in the Agile Periodization framework ${ }^{10}$.

Additional issues with these strategies might be when multiple individuals participate in group sessions, as is the case in team sports. In team sports, one cannot individualize training as this might be the case in individual sports, although separate training sessions aimed at individual needs can be scheduled separately. In team settings, the best starting point might be microloading approach, and saturating certain qualities based on individual and a team needs on particular days or when the opportunity emerges ${ }^{10}$. A further question is what are, and how the hierarchy of qualities should be decided upon? In our opinion, one needs to approach this from an artistic point of view, and combine individual and team approaches, as well as phenomenological, performance, and physiological levels of analysis ${ }^{10}$. It is beyond the scope of the current paper to delve into details of defining qualities, and interested readers are directed to one such approach presented under Agile Periodization framework ${ }^{10}$.

Finally, although this is a theoretical simulation, few takeaways could be implemented in real-life planning. No matter how optimally designed a given training plan is, it can become fragile or even harmful when faced with MCAR and MNAR events. One example might be a personal training client that keeps missing sessions at random or constantly missing lower body resistance workouts on Wednesday. Trainers and coaches are then faced with a conundrum how to gain the most training effect, and how to approach planning in unpredictable circumstances. One potential solution to these problems might be to use the don't break the chain approach (with few variations of the links). For example, a given chain for a personal client doing resistance training might involve the following: Link 1 - Full body session (e.g., bench, military press, chin ups, rows, squats, deadlifts, hip thrusts, core); Link 2 - Lower body session (e.g., back squats, front squats, split squats, deadlifts, hip thrusts); Link 3 - Upper body session (e.g., bench press, chin ups, military press, rows, pull-overs); Link 4 - Arms workout (e.g., biceps, triceps); Link 5 - Easy stretching and a core day. Every time a client misses a session, he or she restarts the chain at the Link 1 - Full body session. To avoid boredom, personal training can create several versions of links (e.g., two or more versions of full body session). On top of that, using Markov model chain might involve set and reps schemes based on individual needs, such as hypertrophy set and reps schemes (e.g., 4x8-12 reps), and strength set and rep schemes (e.g., 5/4/3/2/1), which can provide both variations for every link, but also consider individual needs and preferences. For someone coaching a track and field 
sprinter, who seems to be unreliable with his attendance, one might design the following chain strategy: Link 1 - Acceleration work (10-30m), max speed (using fast-easy-fast method), intensive tempo, squats and bench press; Link 2 - Max speed (60-80m, fast-easy-fast), plyometrics and lower body lifting; Link 3 - Acceleration (10-30m) and upper body lifting; Link 4 - Extensive tempo work and recovery. If this particular athlete keeps missing sessions, the most robust approach would be to implement all the significant methods from Link 1. Furthermore, if this athlete keeps the chain unbroken, he or she will be "rewarded" with Link 4, which represents an easy day and recovery. From Link 4 one can move to Link 2, but again this can be represented with a simple Markov model.

The more complex and elaborate simulation might be needed to test the effects and behavior of this type of planning under normal, MCAR and MNAR conditions. This is due to training load and qualities interactions, adaptation thresholds, nonlinear effects, boredom and other factors that are hard to represent in the simulation model. We speculate that for these reasons the barbell chain strategy did not perform better than other chain strategies. Future simulations and controlled experiments are needed to test this hypothesis.

\section{Conclusions}

Optimal planning strategies might become very fragile under volatile conditions, while robust planning strategies, although less optimal, can remain robust when facing volatile conditions. A few potential planning strategies are presented in the current paper, and they involve the use of randomization, chain strategies, barbell approach to distributing training load and the use of Markov models. To our knowledge, this is the first paper that deals with planning under MCAR events and presents potential solutions by approaching planning from a robust perspective, rather than an optimal perspective.

We would like to finish this paper with the quote by Gerd Gigerenzer: "When faced with significant irreducible uncertainty, the robustness of the approach is more relevant to its future performance than its optimality"

\section{Supplementary Materials}

Supplementary Materials can be found at OSF repository: https://osf.io/et78k/ (DOI 10.17605/OSF.IO/ET78K) Accompanying reproducible R code will be available once this manuscript is accepted for publication in s peer-reviewed journal.

\section{Acknowledgments}

Special thanks to Vladislav Mushinsky, Kevin Kishna, Scot Morrison and Michael McGuigan for providing valuable feedback and corrections. 


\section{References}

1. Bompa TO, Buzzichelli C. Periodization: Theory and Methodology of Training. Sixth edition. Champaign, IL: Human Kinetics; 2019.

2. Bompa TO, Buzzichelli C. Periodization Training for Sports. 3rd ed. edition. Champaign: Human Kinetics Publishers; 2015.

3. DeWeese BH, Hornsby G, Stone M, Stone MH. The training process: Planning for strengthPower training in track and field. Part 2: Practical and applied aspects. 2015;4(4):1-7.

4. DeWeese BH, Hornsby G, Stone M, Stone MH. The training process: Planning for strengthPower training in track and field. Part 1: Theoretical aspects. 2015;4(4):1-10.

5. Deweese B, Gray H, Sams M, K. Scruggs S, Serrano A. Revising the Definition of Periodization: Merging Historical Principles with Modern Concern. Olympic Coach. 2013;Winter:5.

6. Issurin VB. Block Periodization: Breakthrough in Sport Training. (Yessis MA, ed.). Michigan: Ultimate Athlete Concepts; 2008.

7. Issurin VB. Block Periodization 2: Fundamental Concepts and Training Design. 1st edition. Michigan: Ultimate Athlete Concepts; 2013.

8. Issurin VB. Building the Modern Athlete: Scientific Advancements and Training Innovations. 1st edition. Ultimate Athlete Concepts; 2015.

9. Volz KG. Cognitive processes in decisions under risk are not the same as in decisions under uncertainty. July 2012:1-6.

10. Jovanovic M. HIIT High Intensity Interval Training and Agile Periodization. CreateSpace Independent Publishing Platform; 2018.

11. Fisher AJ, Medaglia JD, Jeronimus BF. Lack of group-to-individual generalizability is a threat to human subjects research. Proceedings of the National Academy of Sciences. 2018;115(27):E6106E6115. doi:10.1073/pnas.1711978115

12. Yarkoni T, Westfall J. Choosing Prediction Over Explanation in Psychology: Lessons From Machine Learning. Perspectives on Psychological Science. 2017;12(6):1100-1122. doi:10.1177/1745691617693393

13. Molenaar PCM. A Manifesto on Psychology as Idiographic Science: Bringing the Person Back Into Scientific Psychology, This Time Forever. Measurement: Interdisciplinary Research \& Perspective. 2004;2(4):201-218. doi:10.1207/s15366359mea0204_1

14. Molenaar PC, Campbell CG. The New Person-Specific Paradigm in Psychology. Current Directions in Psychological Science. 2009;18(2):112-117. doi:10.1111/j.1467-8721.2009.01619.x

15. Bosch F. Strength Training and Coordination: An Integrative Approach. 01 edition. 2010 uitgevers; 2015.

16. Kiely J. Periodization, Planning, Prediction: And why the future ain't what it used to be! Techniques Journal of USA Track, Field and Cross-country Association. January 2009. 
17. Kiely J. Letter: New Horizons for the Methodology and Physiology of Training Periodization Block Periodization: New horizon or false dawn? Letter submitted to Sports Medicine. January 2010.

18. Kiely J. New Horizons for the Methodology and Physiology of Training Periodization: Block Periodization: New Horizon or a False Dawn? Sports Medicine. 2010;40(9):803-805. doi:10.2165/11535130-000000000-00000

19. Kiely J. Periodization Paradigms in the 21st Century: Evidence-Led or Tradition-Driven? International Journal of Sports Physiology and Performance. 2012;7(3):242-250. doi:10.1123/ijspp.7.3.242

20. Kiely J. Periodization Theory: Confronting an Inconvenient Truth. Sports Medicine. 2018;48(4):753-764. doi:10.1007/s40279-017-0823-y

21. Kiely J. Planning for physical performance: The individual perspective. In: Performance Psychology. Elsevier; 2011:139-160. doi:10.1016/B978-0-443-06734-1.00010-9

22. Loturco I, Nakamura FY. Training Periodization: An obsolete methodology? Aspetar Journal. 2016;5(1).

23. van Buuren S. Flexible Imputation of Missing Data. 1 edition. Boca Raton, FL: Chapman and Hall/CRC; 2012.

24. Graham JW. Missing Data Analysis: Making It Work in the Real World. Annual Review of Psychology. 2009;60(1):549-576. doi:10.1146/annurev.psych.58.110405.085530

25. Gabbett TJ. The trainingInjury prevention paradox: Should athletes be training smarter and harder? British Journal of Sports Medicine. 2016;50(5):273-280. doi:10.1136/bjsports-2015095788

26. IEEE Standard Glossary of Software Engineering Terminology. IEEE; 1990. doi:10.1109/IEEESTD.1990.101064

27. R Core Team. $R$ : A Language and Environment for Statistical Computing. Vienna, Austria: $\mathrm{R}$ Foundation for Statistical Computing; 2018.

28. Taleb NN. Antifragile: Things That Gain from Disorder. First Edition edition. New York: Random House; 2012.

29. Neth H, Gigerenzer G. Heuristics: Tools for an Uncertain World. In: Scott RA, Kosslyn SM, eds. Emerging Trends in the Social and Behavioral Sciences. Hoboken, NJ, USA: John Wiley \& Sons; 2015:1-18.

30. Phillips N, Neth H, Woike JK, Gaissmaier W. FFTrees: A toolbox to create, visualize, and evaluate fast-and-frugal decision trees. Judgment and Decision Making. 2017;12(4):344-368.

31. DeMiguel V, Garlappi L, Uppal R. Optimal Versus Naive Diversification: How Inefficient is the 1/ NPortfolio Strategy? Review of Financial Studies. 2009;22(5):1915-1953.

32. Gigerenzer G. Striking a Blow for Sanity in Theories of Rationality. In: Models of a Man. MIT Press; 2004:1-12. 
33. Gigerenzer G. Why Heuristics Work. Perspectives on Psychological Science. 2008;3(1):1-10.

34. Gigerenzer G, Gaissmaier W. Heuristic decision making. Annual review of psychology. 2011;62(1):451-482.

35. Gigerenzer G, Hertwig R, Pachur T. Heuristics: The Foundations of Adaptive Behavior. Reprint edition. Oxford University Press; 2015.

36. Gigerenzer G. Risk Savvy: How to Make Good Decisions. Reprint edition. Penguin Books; 2015.

37. Mainik G, Mitov G, Rüschendorf L. Portfolio optimization for heavy-tailed assets: Extreme Risk Index vs. Markowitz. Journal of Empirical Finance. 2015;32:115-134.

38. Mousavi S, Gigerenzer G. Risk, uncertainty, and heuristics. Journal of Business Research. 2014;67(8):1671-1678.

39. Pflug GC, Pichler A, Wozabal D. The $1 / \mathrm{N}$ investment strategy is optimal under high model ambiguity. Journal of Banking and Finance. 2012;36(2):410-417.

40. McGuigan MR. Monitoring Training and Performance in Athletes. 1 edition. Champaign, IL: Human Kinetics; 2017.

41. Williams S, West S, Cross MJ, Stokes KA. Better way to determine the acute:Chronic workload ratio? Br J Sports Med. 2017;51(3):209-210. doi:10.1136/bjsports-2016-096589

42. Jovanovic M. Data Preparation for Injury Prediction. Sport Performance \& Science Reports. August 2018:1.

43. Husson F, Josse J, Pages J. Principal component methods - hierarchical clustering - partitional clustering: Why would we need to choose for visualizing data? September 2010:17.

44. Lê S, Josse J, Husson F. FactoMineR: A Package for Multivariate Analysis. Journal of Statistical Software. 2008;25(1):1-18. doi:10.18637/jss.v025.i01

45. Page SE. The Model Thinker: What You Need to Know to Make Data Work for You. Basic Books; 2018. 